\title{
Synthesis and anti-tubercular activity of Thienyl and Furanyl derivatives
}

\author{
Laura Nogueira de Faria Cardoso ${ }^{1,2}$, Thais Cristina Mendonça Nogueira ${ }^{1,}$, , Carlos Roland Kaiser ${ }^{2}$, \\ James Lewis Wardell ${ }^{1,3}$, Solange Maria Silva Veloso Wardell ${ }^{4}$ and Marcus Vinicius Nora de Souza ${ }^{1,2, *}$ \\ ${ }^{1}$ FioCruz-Fundação Oswaldo Cruz, Instituto de Tecnologia em Fármacos-Farmanguinhos, Rua Sizenando \\ Nabuco, 100, Manguinhos, 21041-250, Rio de Janeiro, RJ, Brazil \\ ${ }^{2}$ Programa de Pós-Graduação em Química, Instituto de Química, Universidade Federal do Rio de Janeiro, \\ Cidade Universitária, CP 68563, 21945-970, Rio de Janeiro, RJ, Brazil \\ ${ }^{3}$ Department of Chemistry, University of Aberdeen, Old Aberdeen, AB24 3UE, Scotland \\ ${ }^{4}$ CHEMSOL, 1 Harcourt Road, Aberdeen, AB15 5NY, Scotland
}

\begin{abstract}
The synthesis and anti-tubercular activity of series of acyl hydrazonyl compounds, namely $(E)-N^{\prime}-$ (arylidene)thiophen-2-carbohydrazide, $(E)$ - $N^{\prime}$-(arylidene)furan-2-carbohydrazide, $(E)$ - $N$-methyl- $N^{\prime}$-arylidenethiophen-2-carbohydrazide, and $E$ )- $N$-methyl- $N^{\prime}$-arylidene-2-(thien-2yl)-acetohydrazide, [32 compounds in all] are reported. The activities of against Mycobacterium tuberculosis H37Rv (ATTC27294) are compared with those $(E)-N^{\prime}$-arylidene-2-(thien-2-yl)-acetohydrazide, previously reported. The most active compounds are (aryl = 5-nitrothien-2-yl), (aryl = 5-nitrofuran-2-yl) and (aryl = 5-nitrothien-2-yl). Moderate activity was displayed by (aryl = 5-nitrofuran-2-yl) and certain derivatives of series where aryl is pyridin-2-yl or an $o$-hydroxyphenyl derivative. Doubling of certain NMR signals of each compound in solution indicates that a mixture of conformers, $Z_{\mathrm{C}(\mathrm{O}) \mathrm{NH}} / E_{(\mathrm{C}=\mathrm{N})}$ and $E_{\mathrm{C}(\mathrm{O}) \mathrm{NH}} / E_{(\mathrm{C}=\mathrm{N})}$ about the $\mathrm{C}(\mathrm{O})-\mathrm{NH}-\mathrm{N}=\mathrm{C}(\mathrm{H}$, aryl) fragment is present. In contrast, only one form for each compound is present in solution from the single set of NMR signals. It is suggested that this form in solution is the $E_{\mathrm{C}(\mathrm{O}) \mathrm{NH}} / E_{(\mathrm{C}=\mathrm{N})}$ form. Only a single set of NMR signals are found for the $N$-methylated derivatives.
\end{abstract}

Keywords: Thienyl derivatives; Furanyl derivatives; Acyl hydrazones.

\section{Introduction}

Thiophene and its derivatives have been well studied as materials, e.g., in applications in organic electronics and photonics ${ }^{1}$ and in the medical area. In the medical area, the thiophene nucleus is present in many natural and synthetic products having a wide range of pharmacological activities, such as antiviral $^{2}$, anticancer ${ }^{3}$, antibacterial ${ }^{4,5}$, antifungal ${ }^{5,6}$, and anti-inflammatory agents ${ }^{7}$.

Our interests in the biological activities and structural chemistry of heterocylic compounds have led us to investigate thiophene and its derivatives, which have been found to exhibit tuberculostatic activity $^{8}$. We have reported the anti-TB activities of acetamido derivatives, 2-( $\left.\mathrm{RR}^{\prime} \mathrm{NCOCH}_{2}\right)$-thiophene $\mathbf{1}^{9-11}$, and more recently acetohydrazide derivatives of thiophene, 2-( $\left.\mathrm{ArCH}=\mathrm{N}-\mathrm{NHCOCH}_{2}\right)$-thiophene $\mathbf{2}^{12}$, see Scheme 1 . In the latter study ${ }^{12}$, the most active compounds were $(2$ : aryl $=5$-nitrothienyl and 5-nitrofuranyl), while among the moderately active compounds were (2: aryl $=$ pyridin-2-yl, or 2-hydroxyphenyl). Due to the promising biological results, we have followed up this study with work on further acylhydrazonyl derivatives of thiophene, namely $(E)-N^{\prime}$-(arylidene)thiophen-2-carbohydrazide 3, (E)- $N$-methyl- $N$ '-arylidene-thiophen-2carbohydrazide, 5, and (E)-N-methyl- $N$ '-arylidene-2(thiophen-2yl)-acetohydrazide $\mathbf{6}$, and in addition, on a series of furanyl compounds, (E)- $N^{\prime}$ (arylidene)furan-2-carbohydrazide 4, see Scheme 1.

As well as reporting the synthesis and biological activities of the new thienyl and furanyl derivatives.

\section{Results and Discussion \\ Chemistry}

The synthesis of the compounds, $\mathbf{3}$ or $\mathbf{4}$, were achieved by reactions of arylaldeydes with 2-( $\mathrm{H}_{2} \mathrm{NNHCO}$-thiophene or furan $), \mathbf{8}$, generated from methyl thiene-2-carboxylate or methyl furan-2carboxylate 7, respectively. Methylation of $2^{12}$ and $\mathbf{3}$ by methyl iodide produced $\mathbf{5}$ and $\mathbf{6}$, respectively, see Scheme 1. 
<smiles>[R]C=NNC(=O)c1ccc(C=C)[pH]1</smiles><smiles>[R]C=NN(C)C(=O)Cc1ccc(C#[R]C=NNC(=O)Cc2cccs2)s1</smiles>

Scheme 1. Reagents and conditions: (i) $\mathrm{SOCl}_{2}, \mathrm{MeOH}, 0^{\circ} \mathrm{C}$-r.t., 24h, 98-100\%; (ii) $\mathrm{N}_{2} \mathrm{H}_{4} \cdot \mathrm{H}_{2} \mathrm{O}(55 \%$ ), EtOH, $80^{\circ} \mathrm{C}, 2-18 \mathrm{~h}, 75-80 \%$; (iii) $\mathrm{EtOH}, \mathrm{RCHO}$, r.t, 1-72h, 40-97\%; (iv) a: acetone, $\mathrm{K}_{2} \mathrm{CO}_{3}$, r.t. 30 min, b: $\mathrm{CH}_{3} \mathrm{I}, 40^{\circ} \mathrm{C}$, 2-24h, 65-89\%.

The choices of the aryl moiety used in this second study were based on the results from our previous study ${ }^{12}$. Thus derivatives containing 2-hydroxyphenyl, 5-nitrothien-2-yl, 5-nitrofuran-2-yl and 2-pyridinyl as the aryl substituents feature strongly in the current report. Table 1 lists the compounds studied and the biological results obtained. All compounds were characterized by IR and NMR spectroscopy.

Table 1. Compounds studied and biological results obtained ${ }^{\mathrm{a}, \mathrm{b}}$.

\begin{tabular}{|c|c|c|c|}
\hline Compound & MIC $(\mu \mathrm{M})$ & Compound & MIC $(\mu \mathrm{M})$ \\
\hline $3 \mathrm{a}\left(\mathrm{R}=5-\mathrm{O}_{2} \mathrm{~N}\right.$-thien-2-yl) & Insol. & 4c $(\mathrm{R}=$ pyridin $-2-\mathrm{yl})$ & Inact. \\
\hline $3 b\left(\mathrm{R}=5-\mathrm{O}_{2} \mathrm{~N}\right.$-furan-2-yl) & Insol. & 4d $\left(\mathrm{R}=2-\mathrm{HOC}_{6} \mathrm{H}_{4}\right)$ & Insol. \\
\hline 3c $(\mathrm{R}=$ =pyridin-2-yl) & 108.2 & $4 \mathbf{e}\left(\mathrm{R}=4-\mathrm{HOC}_{6} \mathrm{H}_{4}\right)$ & Inact. \\
\hline $3 d\left(\mathrm{R}=2-\mathrm{HOC}_{6} \mathrm{H}_{4}\right)$ & Insol. & $\mathbf{4 f}\left(\mathrm{R}=2,3(\mathrm{HO})_{2} \mathrm{C}_{6} \mathrm{H}_{3}\right)$ & Inact. \\
\hline $3 e\left(R=4-H_{0 C} H_{4}\right)$ & 404.8 & $4 \mathrm{~g}\left(\mathrm{R}=2-\mathrm{HO}-4-\mathrm{MeC}_{6} \mathrm{H}_{3}\right)$ & Insol. \\
\hline $3 \mathrm{f}\left(\mathrm{R}=2,4-(\mathrm{HO})_{2} \mathrm{C}_{6} \mathrm{H}_{3}\right)$ & 380.2 & 4h $\left(\mathrm{R}=2-\mathrm{HO}-5-\mathrm{MeC}_{6} \mathrm{H}_{3}\right)$ & Insol. \\
\hline $3 \mathrm{~g}\left(\mathrm{R}=3,4-(\mathrm{HO})_{2} \mathrm{C}_{6} \mathrm{H}_{3}\right)$ & Inact. & $4 \mathbf{i}\left(\mathrm{R}=2-\mathrm{HO}-3-\mathrm{MeOC}_{6} \mathrm{H}_{3}\right)$ & 401.3 \\
\hline $3 \mathrm{~h}\left(\mathrm{R}=2-\mathrm{HO}-4-\mathrm{MeC}_{6} \mathrm{H}_{3}\right)$ & Insol. & $\mathbf{4 j}\left(\mathrm{R}=2-\mathrm{HO}-4-\mathrm{MeOC}_{6} \mathrm{H}_{3}\right)$ & Insol. \\
\hline $3 \mathrm{i}\left(\mathrm{R}=2-\mathrm{HO}-5-\mathrm{MeC}_{6} \mathrm{H}_{3}\right)$ & Inact. & $4 \mathbf{k}\left(\mathrm{R}=4-\mathrm{HO}-3-\mathrm{ClC}_{6} \mathrm{H}_{3}\right)$ & Inact. \\
\hline $3 \mathrm{j}\left(\mathrm{R}=2-\mathrm{HO}-3-\mathrm{MeOC}_{6} \mathrm{H}_{3}\right)$ & 180.5 & 5a $\left(\mathrm{R}=5-\mathrm{O}_{2} \mathrm{~N}\right.$-thien-2-yl $)$ & Insol. \\
\hline $3 \mathrm{k}\left(\mathrm{R}=2-\mathrm{HO}-4 \mathrm{MeOC}_{6} \mathrm{H}_{3}\right)$ & Insol. & $5 \mathbf{b}\left(\mathrm{R}=5-\mathrm{O}_{2} \mathrm{~N}\right.$-furan-2-yl $)$ & Insol. \\
\hline $3 \mathrm{I}\left(\mathrm{R}=2-\mathrm{HO}-3-\mathrm{O}_{2} \mathrm{NC}_{6} \mathrm{H}_{3}\right)$ & 171.2 & 5c $(\mathrm{R}=$ pyridine $-2-\mathrm{yl})$ & 432.9 \\
\hline $3 \mathrm{~m}\left(\mathrm{R}=2-\mathrm{HO}-5-\mathrm{O}_{2} \mathrm{NC}_{6} \mathrm{H}_{3}\right)$ & Insol. & 6a $\left(\mathrm{R}=5-\mathrm{O}_{2} \mathrm{~N}\right.$-thien-2-yl) & 10.5 \\
\hline $3 n\left(\mathrm{R}=4-\mathrm{HO}-3-\mathrm{ClC}_{6} \mathrm{H}_{3}\right)$ & 88.8 & 6b $\left(\mathrm{R}=5-\mathrm{O}_{2} \mathrm{~N}\right.$-furan-2-yl $)$ & 179.2 \\
\hline $4 \mathrm{a}\left(\mathrm{R}=5-\mathrm{O}_{2} \mathrm{~N}\right.$-thien-2-yl) & Insol. & 6c $(\mathrm{R}=$ pyridin $-2-\mathrm{yl})$ & Inact. \\
\hline $4 \mathrm{~b}\left(\mathrm{R}=5-\mathrm{O}_{2} \mathrm{~N}\right.$-furan-2-yl $)$ & 100.3 & 2b $\left(\mathrm{R}=5-\mathrm{O}_{2} \mathrm{~N} \text {-furan-2-yl }\right)^{\mathrm{I} 2}$ & 9.0 \\
\hline $2 \mathrm{a}\left(\mathrm{R}=5-\mathrm{O}_{2} \mathrm{~N} \text {-thien-2-yl) }\right)^{12}$ & 8.5 & & \\
\hline Ethambutol & 15.3 & Isoniazide & 0.46 \\
\hline
\end{tabular}

${ }^{\text {a) }}$ Ins $=$ insoluble; ${ }^{\text {b) }}$ Inact $=$ inactive: $>100 \mu \mathrm{g} / \mathrm{mL}$

It has been variously reported that $N$-acylhydrazones, $\mathrm{RCONHN}=\mathrm{CHR}^{\mathrm{i}}$ can exist in different forms in solution ${ }^{13-18}$. In our previous study ${ }^{11}$ the NMR spectra of compounds 2 indicated the presence of two forms in solution, from the doubling of certain signals. A similar finding was observed for compounds $\mathbf{3}$ in this study. There are, in principle, four possible arrangements about the $\mathrm{C}(\mathrm{O}) \mathrm{NH}=\mathrm{N}$ fragment in acylhydrazones, $\mathrm{RCONHN}=\mathrm{CHR}^{1}$ : these are derived from combinations of two conformers, $E_{\mathrm{C}(\mathrm{O}) \mathrm{NH}} / Z_{\mathrm{C}(\mathrm{O}) \mathrm{NH}}$ (anti-periplanar/syn-periplanar), and two geometric $\mathrm{N}=\mathrm{C}$ isomers $-E_{(\mathrm{C}=\mathrm{N})} / Z_{(\mathrm{C}=\mathrm{N})}$, see Figure 1 . However the $Z_{(\mathrm{C}=\mathrm{N})}$ isomers are generally so much disfavoured for steric reasons, that only $E_{\mathrm{C}(\mathrm{O}) \mathrm{NH}} / E_{(\mathrm{C}=\mathrm{N})}$ and $Z_{\mathrm{C}(\mathrm{O}) \mathrm{NH}} / E_{(\mathrm{C}=\mathrm{N})}$ are observed by NMR spectroscopy. The two conformers are in equilibrium, with different ratios observed in different solutions and temperatures etc. 
<smiles>[R]C=NNC([R])=O</smiles><smiles>[R]C=NNC([R])=O</smiles><smiles>[R]C=NNC([R])=O</smiles><smiles>[R]/C=N\NC([R])=O</smiles>

$\boldsymbol{Z}_{\mathrm{C}(\mathrm{O}) \mathrm{NH}} / \boldsymbol{E}_{(\mathrm{C}=\mathrm{N})}$

$\boldsymbol{E}_{\mathrm{C}(\mathrm{O}) \mathrm{NH}} / \boldsymbol{E}_{(\mathrm{C}=\mathrm{N})}$

$\boldsymbol{Z}_{\mathrm{C}(\mathrm{O}) \mathrm{NH}} / \boldsymbol{Z}_{(\mathrm{C}=\mathrm{N})}$

$E_{\mathrm{C}(\mathrm{O}) \mathrm{NH}} / \boldsymbol{Z}_{(\mathrm{C}=\mathrm{N})}$

Figure 1. Four possible arrangements of $N$-acylhydazones, about the $\mathrm{C}(\mathrm{O}) \mathrm{NHN}=\mathrm{C}$ fragment.

Only one set of NMR signals are observed for the $N$-methylated compounds $\mathbf{5}$ and $\mathbf{6}$. Methyl substitution at nitrogen of the $\mathrm{C}(\mathrm{O}) \mathrm{N}$ fragment results in restricted rotation about the $(\mathrm{O}) \mathrm{C}-\mathrm{N}(\mathrm{Me})$ bond and leads to the less hindered rotamer being strongly favoured, which in the cases of $\mathbf{5}$ and $\mathbf{6}$, is the $\mathrm{Z}_{\mathrm{C}(\mathrm{O}) \mathrm{NMe}}$ conformer. Furthermore, the extra steric hindrance of the methyl substitution would further disadvantage the $\mathrm{Z}_{(\mathrm{N}=\mathrm{C})}$ geometric isomer resulting in formation of just the $\mathrm{E}_{\mathrm{N}=\mathrm{C}} / \mathrm{Z}_{\mathrm{C}(\mathrm{O}) \mathrm{NMe}}$ isomer and elimination of other isomers, at least to the limits of NMR detection.

In contrast to the situation found for the thienyl derivatives $\mathbf{3}$, the analogous furanyl compounds, $\mathbf{4}$, in solution exist in a single conformation as only one set of NMR signals are observed in solution. A similar situation was found for $5-\left(2,5-\mathrm{Cl}_{2} \mathrm{C}_{6} \mathrm{H}_{3}\right)-2-(2-$ $\left.\mathrm{MeOC}_{6} \mathrm{H}_{4} \mathrm{CH}=\mathrm{N}-\mathrm{NHCO}\right)$-furan, $7{ }^{15}$. To find some collaborative support for this finding, we looked at the single crystal X-ray data for compounds $\mathbf{3}$ and $\mathbf{4}$ in the literature.

Various crystal structures for compounds in each of the series $3^{19-21}$ and $4^{22-24}$ have been reported, albeit briefly. As pointed out above, four possible conformations about the $\mathrm{CONH}-\mathrm{N}=\mathrm{N}$ fragment are potentially possible in solution. In the solid state,<smiles>[X]c1ccc(/C=N/NC(=O)c2ccc[pH]2)cc1</smiles>

A<smiles>[X]c1ccc(/C=N/NC(=O)C2=CC=C[IH]2)cc1</smiles>

B rotations about other bonds in the molecule, which are freely allowed in solution, can be frozen out with the result that different conformations are possible. Such additional rotamers, or conformers, arise in the solid state for the furanyl and thienyl derivatives, $\mathbf{3}$ and 4.

Looking at the molecular conformations of all the reported crystal structures of $\mathbf{3}$ and $\mathbf{4}$, four distinct molecular conformations, A-D, are formed, see Figure 2. Forms A and B are one pair of rotamers and $\mathrm{C}$ and $\mathrm{D}$ are another pair of rotamers, arising from rotation about the (thienyll)C-C(O) and (furanyl)C-C(O) bonds, respectively. $\mathrm{A}$ and $\mathrm{B}$ are $Z_{\mathrm{C}(\mathrm{O}) \mathrm{NH}} / E_{(\mathrm{C}=\mathrm{N})}$ forms and $\mathrm{C}$ and $\mathrm{D}$ are $E_{\mathrm{C}(\mathrm{O}) \mathrm{NH}} / E_{(\mathrm{C}=\mathrm{N})}$ forms about the $\mathrm{C}(\mathrm{O})-\mathrm{NH}-\mathrm{N}=\mathrm{CH}$-fragment. The thienyl compounds 3 are present in forms $\mathrm{A}^{19}$, A \& B ${ }^{20}$ or $\mathrm{C}^{21}$, with $\mathrm{A}$ the most common. The finding that both $Z_{\mathrm{C}(\mathrm{O}) \mathrm{NH}} / E_{(\mathrm{C}=\mathrm{N})}$ and $E_{\mathrm{C}(\mathrm{O}) \mathrm{NH}} / E_{(\mathrm{C}=\mathrm{N})}$ molecular structural forms occur for $\mathbf{3}$, is in contrast with the finding for the furanyl compounds, 4 , where only $E_{\mathrm{C}(\mathrm{O}) \mathrm{NH}} / E_{(\mathrm{C}=\mathrm{N})}$ molecular structural forms are found: references for compounds having the $\mathrm{C}$ form, $\mathrm{C} \& \mathrm{D}$ and D forms for solid 4 are 22, 23 are $15 \& 24$, respectively: the most common form for $\mathbf{4}$ is the D form.
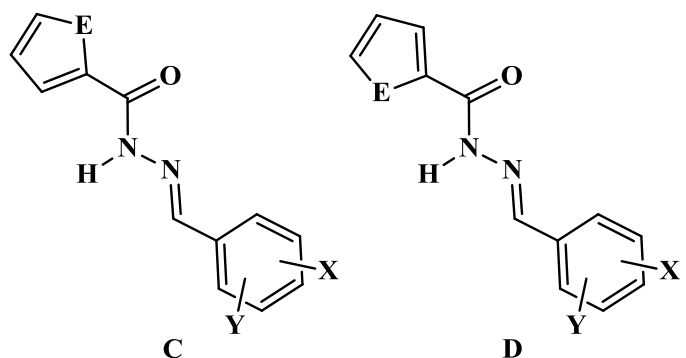

D

Figure 2. The four molecular conformations reported for solid derivatives, $(3: E=S)$ and $(4: E=O)$.

It could be argued that crystal packing effects and intermolecular interactions could so favour the $E_{\mathrm{C}(\mathrm{O}) \mathrm{NH}} / E_{(\mathrm{C}=\mathrm{N})}$ conformers in the solid state, that the solid state findings are not relevant in explaining any solution finding concerning molecular conformations. However the array of compounds $\mathbf{4}$, with mono- or di-substituents, electron releasing or electron withdrawing substituents, small or bulky substituents, strong hydrogen bonding substituents or not, all existing in the $E_{\mathrm{C}(\mathrm{O}) \mathrm{NH}} / E_{(\mathrm{C}=\mathrm{N})}$ conformation is a reasonable pointer to a conclusion that the $E_{\mathrm{C}(\mathrm{O}) \mathrm{NH}} / E_{(\mathrm{C}=\mathrm{N})}$ conformation is very highly favoured over the $Z_{\mathrm{C}(\mathrm{O}) \mathrm{NH}} / E_{(\mathrm{C}=\mathrm{N})}$ conformation for furanyl derivatives 4 .

\section{Biological activity}

Table 1 lists the results of biological activity against M. tuberculosis H37Rv (ATTC27294). Of the 30 or so compounds prepared, 13 were not sufficiently soluble for the screening and 8 were deemed inactive. The most active compounds were the 5-nitrothienyl derivatives, $\mathbf{6 a}$ and $\mathbf{2} \mathbf{a}^{12}$ and the 5nitrofuranyl derivative $\mathbf{2} \mathbf{b}^{12}$ with $\mathrm{MIC}(\mu \mathrm{M})$ values of $10.8,8.5$ and 9.0 , respectively, respectively, compared to those of ethambutol of 15.3 and isoniazide of 0.46 . The other two 5-nitrothienyl derivatives, $\mathbf{3 a}$ and $\mathbf{4 a}$ were insufficiently soluble for the screening, thereby preventing a comparison involving all the 5-nitrothienyl compounds. 
Moderate activity was determined for the 5nitrofuranyl derivatives, $\mathbf{4 b}$ and $\mathbf{6 b}$ : again a comparison of all the 5-nitrofuranyl derivatives was thwarted by the lack of the adequate solubility of $\mathbf{3 b}$.

Of the remainder of the compounds, moderate activity was also found for $\mathbf{3 c}$ and $\mathbf{3 n}$ : similar moderate activities were found for the corresponding $\mathrm{RCH}=\mathrm{NNHCOCH}_{2}$-thiophenederivatives, 2, in the earlier study ${ }^{12}$. The activities determined for the various hydroxyphenyl derivatives, $\mathbf{3 d - 3 n}$ and $\mathbf{4 d - 4 k}$ are so mixed that no general trend can be extracted from the data, apart from the generally lower activity of the furanyl compounds 4 , compared to those of the analogous thienyl compounds $\mathbf{3}$. The activity of the 5-nitro-heterocyclic compounds, $\mathbf{2 a}, \mathbf{2 b}$ and $\mathbf{6 a}$, good on one hand, and the 2-hydroxyphenyl and pyridine-2-ylderivatives, moderately so on the other, does point to differing modes of action. The latter compounds, for example are potentially good chelators of metal ions, while the former compounds do not possess this property, but could be sources of free radicals via reduction of the nitro groups ${ }^{12}$ and references therein.

\section{Conclusion}

The most active compounds against $M$. tuberculosis H37Rv (ATTC27294) are (2a: aryl = 5-nitrothien-2-yl), (2b: aryl = 5-nitrofuran-2-yl) and (6: aryl $=5$-nitrothien-2-yl). Moderate activity was displayed by (4b: aryl = 5-nitrofuran-2-yl) and certain derivatives of series $\mathbf{3}$ where aryl is an $o$-hydroxyphenyl derivative or pyridin-2-yl. The screening data from this study concurs in the main over substituent effects with our earlier findings ${ }^{11}$. Each compound $\mathbf{3}$ in solution exists as a mixture of conformers, $Z_{\mathrm{C}(\mathrm{O}) \mathrm{NH}} / E_{(\mathrm{C}=\mathrm{N})}$ and $E_{\mathrm{C}(\mathrm{O}) \mathrm{NH}} / E_{(\mathrm{C}=\mathrm{N})}$ about the $\mathrm{C}(\mathrm{O})-\mathrm{NH}-\mathrm{N}=\mathrm{C}(\mathrm{H}$, aryl $)$ fragment. In contrast, only one form, $E_{\mathrm{C}(\mathrm{O}) \mathrm{NH}} / E_{(\mathrm{C}=\mathrm{N})}$, for each compound $\mathbf{4}$ is present in solution.

\section{Acknowledgements}

The authors would like to acknowledge CNPq and Fiocruz for financial and infrastructure assistance.

\section{Experimental Section \\ Chemistry}

Melting points were determined on a Buchi apparatus and are uncorrected. Infrared spectra were recorded on a Thermo Nicolet Nexus 670 spectrometer in potassium bromide pellets. HRMS were performd on Bruker Compact QTOF mass spectrometer system. NMR spectra were recorded on a Bruker Avance 400 or 500 spectrometers in ${ }^{D M S O}-\mathrm{d}_{6}$ at room temperature. For TLC plates coated with silica gel were run in hexane/acetate mixture and spots were developed in ultraviolet and solution of ninhydrine $(0.2 \% \mathrm{p} / \mathrm{v}$ in ethanol). Compounds 7 were prepared as reported ${ }^{12}$.
Synthesis of methyl thiophene-2-carboxylate (7: E $=S$ ) and methyl furan-2-carboxylate $(7: E=O)$

Thionyl chloride $(8.25 \mathrm{~mL}, 117.2 \mathrm{mmol})$ was slowly added to methanol $(80.0 \mathrm{~mL})$ at $0{ }^{\circ} \mathrm{C}$ under nitrogen atmosphere. After stirring for 20 minutes, 2thiophenecarboxylic acid or 2-furancarboxylic acid (3.0 g, $23.5 \mathrm{mmol})$ was added. The mixture was stirred at room temperature for 24 hours, concentrated under reduced pressure, and the residue was neutralized with saturated aqueous solution of sodium bicarbonate. The layers were separated and the aqueous phase was extracted with dichloromethane ( $3 \times 15 \mathrm{~mL})$. The organic phases were combined, dried over magnesium sulfate and concentrated under vacuum to yield $\mathbf{7}$ as yellow syrup (3.26 g, 98-100\%). The crude product was used without further purification. The product (7: $\mathrm{E}=\mathrm{O}$ ) was particularly unstable and the NMR spectra were not obtained.

\section{Compound (7: $\mathbf{E}=\mathbf{S})$}

Yield: 98\%; yellow syrup.

${ }^{1}$ H NMR (400 MHz; DMSO)反: $7.96\left(1 \mathrm{H} ; \mathrm{dd} ; J_{H H}=\right.$ 5.0 and $1.2 \mathrm{~Hz}$; H-5), $7.82\left(1 \mathrm{H}\right.$; dd; $J_{H H}=3.7$ and 1.2 $\mathrm{Hz}$; H-3), $7.23\left(1 \mathrm{H}\right.$; dd; $J_{H H}=3.8$ and $\left.4.9 \mathrm{~Hz} ; \mathrm{H}-4\right)$, 3.83 (3H; s; $\left.\mathrm{OCH}_{3}\right) .{ }^{13} \mathbf{C}$ NMR(100 MHz DMSO) $\delta$ : 161.9 (C=O), 133.9 (C-2), 133.7 (C-3), 132.7 (C-4), 128.3 (C-5), $52.1\left(\mathrm{OCH}_{3}\right)$.

HRMS $\boldsymbol{m} / \boldsymbol{z}: 164.9990 \quad[\mathrm{M}+\mathrm{Na}]^{+}$(calcd for $\left[\mathrm{C}_{6} \mathrm{H}_{6} \mathrm{O}_{2} \mathrm{~S}+\mathrm{Na}\right]^{+}:$164.9986).

IR $v_{\max }\left(\mathbf{c m}^{-1}\right.$; KBr pellets): $1712(\mathrm{C}=\mathrm{O}) ; 1265$ $\left(\mathrm{O}-\mathrm{CH}_{3}\right)$.

Synthesis of thien-2-yl-2-carbohydrazide $(8: E=S)$ and furan-2-ylcarbohydrazide $(8: E=O)$

To a solution of (7) $(0.5 \mathrm{~g}, 3.4 \mathrm{mmol})$ in ethanol (5.0 $\mathrm{mL})$ was added hydrazine hydrate $(0.46 \mathrm{~mL}, 5.2$ mmol, aqueous solution 55\%). After stirring for 18 hours at $80^{\circ} \mathrm{C}$, the reaction mixture was concentrated under reduced pressure, and the residue was washed with cold ethanol $(2 \times 10.0 \mathrm{~mL})$, followed by diethyl ether $(2 \times 10.0 \mathrm{~mL})$, and dried. Compounds 8 were obtained as solids, in yields of $c a 80 \%$.

\section{Compound (8: $\mathbf{E}=\mathbf{S}$ )}

Yield: $80 \%$; white solid; m.p. $136-137^{\circ} \mathrm{C}$.

${ }^{1}$ H NMR (400 MHz; DMSO) $\delta: 9.75(1 \mathrm{H} ; \mathrm{sl} ; \mathrm{NH})$, $7.74\left(1 \mathrm{H} ; \mathrm{dd} ; J_{H H}=5.0\right.$ and $\left.0.9 \mathrm{~Hz} ; \mathrm{H}-5\right), 7.71(1 \mathrm{H}$; $\mathrm{dd} ; J_{H H}=3.7$ and $\left.0.9 \mathrm{~Hz} ; \mathrm{H}-3\right), 7.13\left(1 \mathrm{H} ; \mathrm{t} ; J_{H H}=3.8\right.$ $\mathrm{Hz}), 4.57$ (2H; sl; $\left.\mathrm{NH}_{2}\right)$.

IR $v_{\max }\left(\mathbf{c m}^{-1} ; \mathrm{KBr}\right.$ pellets $): 1622(\mathrm{C}=\mathrm{O}) ; 3100$ $\left(\mathrm{NH}_{2}\right)$.

Compound (8: $\mathrm{E}=\mathbf{O}$ )

Yield: $80 \%$; light yellow solid;

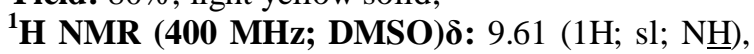
$7.80\left(1 \mathrm{H} ; \mathrm{d} ; J_{H H}=1.7 \mathrm{~Hz} ; \mathrm{H}-5\right), 7.07(1 \mathrm{H} ; \mathrm{d}$; $\left.J_{H H}=3.4 \mathrm{HZ} ; \mathrm{H}-3\right), 6.59\left(1 \mathrm{H} ; \mathrm{dd} ; J_{H H}=3.4\right.$ and 1.7 $\mathrm{Hz} ; \quad \mathrm{H}-4), \quad 4.41 \quad\left(2 \mathrm{H} ; \quad \mathrm{sl} ; \quad \mathrm{N}_{2}\right)$. 
${ }^{13}$ C NMR(100 MHz DMSO) $\delta$ : $157.8(\mathrm{C}=\mathrm{O}), 146.9$ (C-2), 144.8 (C-5), 112.6 (C-4), 111.4 (C-3).

HRMS $m / z: 149.0330 \quad[\mathrm{M}+\mathrm{Na}]^{+}$(calcd for $\left[\mathrm{C}_{5} \mathrm{H}_{6} \mathrm{~N}_{2} \mathrm{O}_{2}+\mathrm{Na}\right]^{+}:$149,0327).

IR $v_{\max }\left(\mathbf{c m}^{-1} ; \mathbf{K B r}\right.$ pellets): $1683(\mathrm{C}=\mathrm{O}) ; 3145$ $\left(\mathrm{NH}_{2}\right)$.

\section{General procedures for}

(E)-N'-benzylidenethiophene-2-carbohydrazide (3) or (E)- $N{ }^{\star}$-benzylidenefuran-2-carbohydrazide (4).

(E)- $N$ '-Benzylidenethiophene-2-carbohydrazide (3) or (E)- $N$ '-benzylidenefuran-2-carbohydrazide (4) derivatives were prepared by reaction between the appropriate carbohydrazide $8(0.2 \mathrm{~g}, 1.4 \mathrm{mmol})$ and the appropriate arenecarbaldehyde $(1.6 \mathrm{mmol})$ in ethanol $(2.0 \mathrm{ml})$. The reaction mixture was stirred for 1-72 hours at room temperature. After that, the excess of solvent was concentrated under reduced pressure and the residue was purified by washing with cold diethyl ether $(2 \times 10.0 \mathrm{~mL})$, leading to the pure derivatives 3a-n and 4a-k as solids in 40-97\% yields.

\section{$(E)-N^{\prime}-[(5-N i t r o t h i o p h e n-2-$}

yl)methylene]thiophene-2-carbohydrazide (3a)

Yield: $80 \%$; orange solid; m.p. $268-269^{\circ} \mathrm{C}$.

${ }^{1}$ H NMR (400 MHz; DMSO) $\delta: 12.24(1 \mathrm{H} ; \mathrm{sl} ; \mathrm{NH})$, $8.30\left(1 \mathrm{H} ; \mathrm{s} ; \mathrm{H}-4\right.$ '), $8.13\left(1 \mathrm{H} ; \mathrm{d} ; J_{H H}=4.0 \mathrm{~Hz}, \mathrm{H}-3\right)$, 8.04-7.93 (2H; m; H-8' and $\left.\mathrm{H}-9^{\prime}\right), 7.58\left(1 \mathrm{H} ; \mathrm{d} ; J_{H H}=\right.$ $4.2 \mathrm{~Hz} \mathrm{H}-5), 7.25\left(1 \mathrm{H} ; \mathrm{d} ; J_{H H}=4.3 \mathrm{~Hz} ; \mathrm{H}-4\right)$. ${ }^{13} \mathrm{C}$ NMR(100 MHz DMSO) $\delta$ : $161.3(\mathrm{C}=\mathrm{O}), 150.9$ (C-4'), 146.3 (C-2), 140.9 (C-5'), 137.0 (C-7'), 135.5 (C-5), 132.5 (C-4), 130.5 (C-3), 129.7 (C-8'), $128.2\left(\mathrm{C}-9^{\prime}\right)$.

HRMS $\boldsymbol{m} / \boldsymbol{z}: 303.9837 \quad[\mathrm{M}+\mathrm{Na}]^{+}$(calcd for $\left[\mathrm{C}_{10} \mathrm{H}_{7} \mathrm{~N}_{3} \mathrm{O}_{3} \mathrm{~S}_{2}+\mathrm{Na}\right]^{+}$: 303,9827).

IR $v_{\max }\left(\mathbf{c m}^{-1}\right.$; KBr pellets): $1649(\mathrm{C}=\mathrm{O}) ; 3101$ $(\mathrm{N}-\mathrm{H})$.

$(E)-N$-((5-Nitrofuran-2-yl)methylene)thiophene2-carbohydrazide (3b)

Yield: $92 \%$; yellow solid; m.p. $255-256^{\circ} \mathrm{C}$.

${ }^{1}$ H NMR (400 MHz; DMSO) $\boldsymbol{\delta}: 12.26(1 \mathrm{H} ; \mathrm{sl} ; \mathrm{NH})$, 8.10-7.96 (3H; m; H-4'; H-8' and H-9'), $7.81(1 \mathrm{H}$; d; $\left.J_{H H}=3.9 \mathrm{~Hz} ; \mathrm{H}-5\right), 7.28\left(1 \mathrm{H} ; \mathrm{d} ; J_{H H}=3.9 \mathrm{~Hz} ; \mathrm{H}-4\right)$, 7.26-7.24 (1H; m; H-3). ${ }^{13} \mathbf{C}$ NMR(100 MHz DMSO) 8: $161.6(\mathrm{C}=\mathrm{O}), 157.9(\mathrm{C}-2), 151.6\left(\mathrm{C}-4^{\prime}\right)$, 137.5 (C-5'), 135.2 (C-3), 132.7 (C-2) 131.4 (C-7'), 129.7 (C-8'), 128.2 (C-9'), 127.1 (C-9').

HRMS $m / z: 288.0082 \quad[\mathrm{M}+\mathrm{Na}]^{+}$(calcd for $\left[\mathrm{C}_{10} \mathrm{H}_{7} \mathrm{~N}_{3} \mathrm{O}_{4} \mathrm{~S}+\mathrm{Na}\right]^{+}:$:288,0055).

IR $v_{\max }\left(\mathbf{c m}^{-1}\right.$; $\mathbf{K B r}$ pellets): $1629(\mathrm{C}=\mathrm{O}) ; 3209$ $(\mathrm{N}-\mathrm{H})$.

(E)- $N$ '-(Pyridin-2-ylmethylene)thiophene-2carbohydrazide (3c)

Yield: $74 \%$; pale yellow syrup; m.p. $149-150^{\circ} \mathrm{C}$.

${ }^{1} \mathbf{H}$ NMR (400 MHz; DMSO)反: $12.05(1 \mathrm{H} ; \mathrm{sl} ; \mathrm{NH})$, $8.64\left(1 \mathrm{H} ; \mathrm{d} ; J_{H H}=4.7 \mathrm{~Hz} ; \mathrm{H}^{-} 7^{\prime}\right), 8.47\left(1 \mathrm{H} ; \mathrm{s} ; \mathrm{H}-4^{\prime}\right)$, 8.17-7.93 (4H; m; H-5; H-8'; H-9' and H-10'), 7.44 $\left(1 \mathrm{H} ; \operatorname{dd} J_{H H}=6.5\right.$ and $\left.5.2 \mathrm{~Hz} ; \mathrm{H}-3\right), 7.25(1 \mathrm{H}$; $\left.\mathrm{t} ; J_{H H}=4.4 \mathrm{~Hz} \mathrm{H}-4\right) .{ }^{13} \mathbf{C}$ NMR(100 MHz DMSO) ठ: $161.5(\mathrm{C}=\mathrm{O}), 152.9$ (C-4'), 149.4 (C-7'), 147.4 (C-2), 137.1 (C-9'), 135.0 (C-5), 132.3 (C-4), 129.3 (C-3), 128.2 (C-5'), 126.8 (C-10') 124.4 (C-8'). HRMS $m / z: 254.0382 \quad[\mathrm{M}+\mathrm{Na}]^{+}$(calcd for $\left[\mathrm{C}_{11} \mathrm{H}_{9} \mathrm{~N}_{3} \mathrm{OS}+\mathrm{Na}\right]^{+}:$254,0364). IR $\mathbf{v}_{\max }\left(\mathbf{c m}^{-1} ; \mathbf{~ K B r}\right.$ pellets): $1649(\mathrm{C}=\mathrm{O}) ; 3101(\mathrm{~N}-\mathrm{H})$.

(E)- $N$ '-(2-Hydroxybenzylidene)thiophene-2carbohydrazide (3d)

Yield: $75 \%$; light yellow solid; m.p. $168-169^{\circ} \mathrm{C}$.

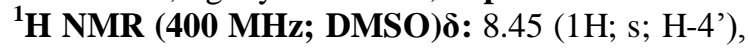
8.33 (1H; s; H-4'), 7.32-7.24 (3H; m; H-7', H-8' and H-9'), $7.57\left(1 \mathrm{H} ; \mathrm{d} ; J_{H H}=7.4 \mathrm{~Hz} ; \mathrm{H}-10^{\prime}\right), 6.99-6.91$ (3H; m; H-3, H-4 and H-5). ${ }^{13} \mathbf{C}$ NMR(100 MHz DMSO) $\delta: 162.8 \& 161.2(\mathrm{C}=\mathrm{O}), 157.6 \& 157.4$ (C-6'), $147.8 \& 140.8$ (C-4'), 137.7 (C-2), 134.8 \& 134.6 (C-5), $133.2 \& 132.1$ (C-4), $131.4 \& 130.9$ (C-3), $129.2 \& 128.2$ (C-10'), 126.6 \& 126.1 (C-8'), 120,5 (C-5'), $119.6 \& 119.4$ (C-9'), $116.6 \& 116.4$ (C-7').

HRMS $\boldsymbol{m} / \boldsymbol{z}: 269.0382 \quad[\mathrm{M}+\mathrm{Na}]^{+}$(calcd for $\left.\left[\mathrm{C}_{12} \mathrm{H}_{10} \mathrm{~N}_{2} \mathrm{O}_{2} \mathrm{~S}+\mathrm{Na}\right]^{+}: 269,0361\right) . \mathbf{I R} \mathbf{v}_{\max }\left(\mathbf{c m}^{-1}\right.$; KBr pellets): $1658(\mathrm{C}=\mathrm{O}) ; 3265(\mathrm{~N}-\mathrm{H})$.

(E)- $N$ '-(4-Hydroxybenzylidene)thiophene-2carbohydrazide (3e)

Yield: 62\%; light yellow solid; m.p. $256-257^{\circ} \mathrm{C}$. ${ }^{1}$ H NMR (400 MHz; DMSO) 8 : $11.72(1 \mathrm{H}$; sl; NH), 8.54 (1H; s; H-4'), 8.36 (1H; s; H-4'), 8.05-7.84 (5H; m; H-6', H-10' and $\mathrm{OH}), 7.74-7.57$ (5H; m; H-7', $\mathrm{H}-9$ ' and $\mathrm{OH}), 7.22\left(2 \mathrm{H} ; \mathrm{t} ; J_{H H}=8.7 \mathrm{~Hz} ; \mathrm{H}-5\right), 6.88$ $\left(4 \mathrm{H} ; \mathrm{d} ; J_{H H}=6.2 \mathrm{~Hz} ; \mathrm{H}-3\right.$ and H-4). ${ }^{13} \mathbf{C} \mathbf{N M R}(\mathbf{1 0 0}$ MHz DMSO) 8: $161.1 \& 160.4(\mathrm{C}=\mathrm{O}), 160.3 \&$ 159.4 (C-4'), $160.4 \& 148.0$ (C-8'), $144.3 \& 139.5$ (C-2), $134.8 \& 134.5$ (C-5), $133.3 \& 131.5$ (C-4), $130.1 \& 129.0$ (C-3), $128.7 \& 128.1$ (C-5'), 126.6 \& 125.2 (C-6' and C-10'), $115.9 \& 115.8$ (C-7' and C-9').

HRMS $\boldsymbol{m} / \boldsymbol{z}: 269.0364 \quad[\mathrm{M}+\mathrm{Na}]^{+} \quad$ (calcd for $\left.\left[\mathrm{C}_{12} \mathrm{H}_{10} \mathrm{~N}_{2} \mathrm{O}_{2} \mathrm{~S}+\mathrm{Na}\right]^{+}: 269,0361\right) . \mathbf{I R} \mathbf{v}_{\max }\left(\mathbf{c m}^{-1} ; \mathbf{K B r}\right.$ pellets): $1624(\mathrm{C}=\mathrm{O}) ; 3236(\mathrm{~N}-\mathrm{H})$.

(E)- $N^{\prime}$-(2,4-Dihydroxybenzylidene)thiophene-2carbohydrazide (3f)

Yield: $67 \%$; light yellow solid; m.p. $239-240^{\circ} \mathrm{C}$.

${ }^{1}$ H NMR (400 MHz; DMSO)d: 10.83 (4H; sl; $\mathrm{NH}$ and $\mathrm{OH}), 8.49\left(1 \mathrm{H} ; \mathrm{s} ; \mathrm{H}-4^{\prime}\right), 7.89-7.86(2 \mathrm{H} ; \mathrm{m}$; H-9', H-10'), $7.33\left(1 \mathrm{H} ; \mathrm{d} ; J_{H H}=8.4 \mathrm{~Hz} ; \mathrm{H}^{\prime} 7^{\prime}\right)$, 6.37-6.32 (3H; m; H-3, H-5 and H-5). ${ }^{13} \mathbf{C}$ NMR(100 MHz DMSO) 8: $162.1 \& 161.7(\mathrm{C}=\mathrm{O}), 160.8$ \& 160.7 (C-6'), $159.4 \& 157.2$ (C-8'), $148.6 \& 141.4$ (C-4'), $134.5 \& 134.2$ (C-10'), 131.8 \& 131.1 (C-2), $128.8 \& 128.2$ (C-3 and C-4), $127.5 \& 126.5$ (C-5), $110.6 \& 110.2$ (C-5'), $108.2 \& 108.1$ (C-9'), 102.6 \& 102.5 (C-7').

HRMS $\boldsymbol{m} / \boldsymbol{z}: 285.0332 \quad[\mathrm{M}+\mathrm{Na}]^{+}$(calcd for $\left.\left[\mathrm{C}_{12} \mathrm{H}_{10} \mathrm{~N}_{2} \mathrm{O}_{3} \mathrm{~S}+\mathrm{Na}\right]^{+}: 285,0310\right)$.

IR $v_{\max }\left(\mathbf{c m}^{-1} ; \mathbf{K B r}\right.$ pellets $): 1641(\mathrm{C}=\mathrm{O}) ; 3120$ $(\mathrm{N}-\mathrm{H})$ 
(E)- $N^{\prime}$-(3,4-Dihydroxybenzylidene)thiophene-2carbohydrazide (3g)

Yield: 97\%; yellow solid; m.p. $226-227^{\circ} \mathrm{C}$. ${ }^{1}$ H NMR (400 MHz; DMSO)反: 11.64 (1H; sl; NH), 11.59 (1H; sl; NH), 8.24 (1H; s; H-4'), 8.03 (1H; s; H-4'), 7.95-7.93 (2H; m; H-4 and H-5), $7.86(2 \mathrm{H}$; $\mathrm{dd} ; J_{H H}=10.5$ and $4.3 \mathrm{~Hz}$; H-4 and H-5), $7.29(1 \mathrm{H}$; s; H-6'), 7.24-7.21 (3H; m; H-6', H-9' and H-10'), 6.96 $\left(2 \mathrm{H}\right.$; dd; $J_{H H}=12.5$ and $7.9 \mathrm{~Hz} ; \mathrm{H}^{\prime} 9^{\prime}$ and $\left.\mathrm{H}-10 '\right)$, $6.81\left(2 \mathrm{H} ; \mathrm{d} ; J_{H H}=5.2 ; \mathrm{H}-3\right) .{ }^{13} \mathbf{C} \mathbf{N M R}(100 \mathbf{M H z}$ DMSO) $\delta: 161.0 \& 160.5(\mathrm{C}=\mathrm{O}), 148.9 \& 148.2$ (C-4' and C-7'), $134.7 \& 134.4$ (C-8'), 131.5 (C-5), 128.6 (C-3), 128.1 (C-4), $126.6 \& 125.1$ (C-5'), $121.9 \& 120.6$ (C-10'), 115.7 \& 113.9 (C-6'), 113.2 \& 112.7 (C-9').

HRMS $\boldsymbol{m} / \boldsymbol{z}: 285.0334 \quad[\mathrm{M}+\mathrm{Na}]^{+}$(calcd for $\left[\mathrm{C}_{12} \mathrm{H}_{10} \mathrm{~N}_{2} \mathrm{O}_{3} \mathrm{~S}+\mathrm{Na}\right]^{+}:$285,0310).

IR $v_{\max }\left(\mathbf{c m}^{-1} ; \mathbf{K B r}\right.$ pellets $): 1641(\mathrm{C}=\mathrm{O}) ; 3265$ $(\mathrm{N}-\mathrm{H})$.

(E)- $N^{\prime}$-(2-Hydroxy-4-methylbenzylidene)thiophene-2-carbohydrazide (3h) Yield: $74 \%$; yellow solid; m.p. $125-126^{\circ} \mathrm{C}$.

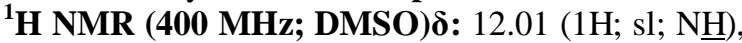
$8.87\left(1 \mathrm{H} ; \mathrm{s} ; \mathrm{H}-7^{\prime}\right), 8.55(1 \mathrm{H} ; \mathrm{s} ; \mathrm{H}-4$ '), $7.91-7.88$ $\left(2 \mathrm{H} ; \mathrm{m}\right.$; H-9' and $\left.\mathrm{H}-10^{\prime}\right), 6.59-6.51(3 \mathrm{H} ; \mathrm{m} ; \mathrm{H}-3$, $\mathrm{H}-4$ and $\mathrm{H}-5), 3.78\left(3 \mathrm{H} ; \mathrm{s} ; \mathrm{CH}_{3}\right) .{ }^{13} \mathbf{C}$ NMR(100 MHz DMSO) $\delta: 163.4 \& 162.1(\mathrm{C}=\mathrm{O}), 162.0 \&$ 160.7 (C-6'), 159.3 \& 157.3 (C-4'), $148.4 \& 141.0$ (C-2), $137.7 \& 132.7$ (C-3), $134.5 \& 131.7$ (C-5), $131.9 \& 130.9$ (C-4), $129.0 \& 128.2$ (C-8'), $127.4 \&$ 126.6 (C-10'), $111.8 \& 111.4$ (C-5'), $107.1 \& 106.5$ (C-9'), $101.2 \& 101.0$ (C-7'), $55.4 \& 55.3\left(\mathrm{CH}_{3}\right)$.

HRMS $\boldsymbol{m} / \boldsymbol{z}: 299.0492 \quad[\mathrm{M}+\mathrm{K}]^{+}$(calcd for $\left[\mathrm{C}_{13} \mathrm{H}_{12} \mathrm{~N}_{2} \mathrm{O}_{2} \mathrm{~S}+\mathrm{K}\right]^{+}:$299,0257).

IR $v_{\max }\left(\mathbf{c m}^{-1}\right.$; KBr pellets): $1629(\mathrm{C}=\mathrm{O}) ; 3446$ $(\mathrm{N}-\mathrm{H})$.

(E)-N'-(2-Hydroxy-5-methyl-

benzylidene)thiophene-2-carbohydrazide (3i)

Yield: $66 \%$; yellow solid; m.p. $268-269^{\circ} \mathrm{C}$

${ }^{1}$ H NMR (400 MHz; DMSO)反: 12.07 (1H; sl; NH), 8.93 (1H; s; H-10'), 8.58 (1H; s; H-4'), 7.92-7.86 $(3 \mathrm{H}$; m; H-5; H-7' and H-8'), 7.25-7.20 (2H; m; H-3 and $\mathrm{H}-4), 2.26\left(3 \mathrm{H} ; \mathrm{s} ; \mathrm{CH}_{3}\right) .{ }^{13} \mathbf{C}$ NMR(100 MHz DMSO) $\delta: 162.5(\mathrm{C}=\mathrm{O}), 156.5 \& 155.2\left(\mathrm{C}-6^{\prime}\right)$, 157.5 (C-4'), 147.7 (C-2), 137.7 (C-3), 133.9 (C-5), 132.1 (C-4), $131.7 \& 130.5$ (C-10'), $129.2 \& 129.1$ (C-9'), $128.2 \& 127.9$ (C-8'), 118.4 \& 117.9 (C-5'), $116.4 \& 116.2$ (C-7'), $19.9\left(\mathrm{CH}_{3}\right)$.

HRMS $\boldsymbol{m} / \boldsymbol{z}: \quad 283.0542 \quad[\mathrm{M}+\mathrm{Na}]^{+}$(calcd for $\left[\mathrm{C}_{13} \mathrm{H}_{12} \mathrm{~N}_{2} \mathrm{O}_{2} \mathrm{~S}+\mathrm{Na}\right]^{+}:$283,0517).

IR $v_{\max }\left(\mathbf{c m}^{-1}\right.$; KBr pellets): $1627(\mathrm{C}=\mathrm{O}) ; 3454$ $(\mathrm{N}-\mathrm{H})$.

(E)-N'-(2-Hydroxy-3-methoxy-

benzylidene)thiophene-2-carbohydrazide (3j)

Yield: $96 \%$; yellow solid; m.p. $143-144^{\circ} \mathrm{C}$.

${ }^{1}$ H NMR (400 MHz; DMSO)d: $12.10(1 \mathrm{H} ; \mathrm{sl}$; NH), 11.79 (1H; sl; NH), 8.64 (1H; s; H-4'), 8.47 (1H; s; H-4'), 7.93-7.90 (2H; m; H-9' and H-10'), 7.26-7.17 (3H; m; H-8', H-9' and H-10'), 7.05-7.00 (2H; m; H-5 and H-8'), 6.89-6.85 (2H, m, H-3 and H-4), 3.82 $\left(3 \mathrm{H} ; \mathrm{s} ; \mathrm{OCH}_{3}\right) .{ }^{13} \mathbf{C} \quad \mathbf{N M R}(\mathbf{1 0 0} \quad \mathbf{M H z}$ DMSO)

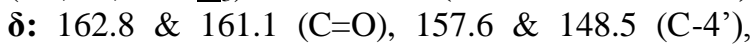
$148.5 \& 147.6$ (C-2), $147.1 \& 146.1$ (C-7'), 140.8 \& 137.7 (C-6'), $134.6 \& 133.2$ (C-3), $132.2 \& 129.2$ (C-5), $128.3 \& 126.7$ (C-4), $122.1 \& 120.9$ (C-5'), $119.1 \& 118.4$ (C-10'), 117.6 \& 115.3 (C-9'), 113.8 \& 112.9 (C-8'), $55.7\left(\mathrm{OCH}_{3}\right)$.

HRMS $\boldsymbol{m} / \boldsymbol{z}$ : $299.0482[\mathrm{M}+\mathrm{Na}]^{+}$(calcd for $\left.\mathrm{C}_{13} \mathrm{H}_{12} \mathrm{~N}_{2} \mathrm{O}_{3} \mathrm{~S}+\mathrm{Na}\right]^{+}:$299,0466)

IR $v_{\max }\left(\mathbf{c m}^{-1}\right.$; KBr pellets $): 1641(\mathrm{C}=\mathrm{O}) ; 3554$ $(\mathrm{N}-\mathrm{H})$.

(E)-N'-(2-Hydroxy-4-methoxy-

benzylidene)thiophene-2-carbohydrazide (3k)

Yield: $84 \%$; yellow solid; m.p. $151-152^{\circ} \mathrm{C}$.

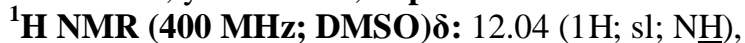

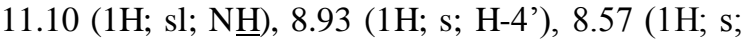
H-4'), 8.03-7.69 (4H; m; H-9' and H-10'), $7.23(2 \mathrm{H}$; s; H-7'), 6.81-6.74 (3H; m; H-3, H-4 and H-5), 2.28 $\left(6 \mathrm{H} ; \mathrm{s} ; \mathrm{CH}_{3}\right) .{ }^{13} \mathbf{C}$ NMR(100 MHz DMSO) $\delta: 162.6$ $(\mathrm{C}=\mathrm{O}), 157.5$ (C-2), $157.4 \& 156.5$ (C-6'), $148.1 \&$ 141.8 (C-4'), 141.4 \& 141.1 (C-8'), 134.6 (C-3), 132.0 (C-5), $129.4 \& 129.1$ (C-10’), 128.2 (C-4), $126.6 \& 126.1$ (C-5') $120.4 \& 116.8$ (C-9'), 117.8 \& 116.1 (C-7'), $21.3 \& 21.2\left(\mathrm{CH}_{3}\right)$.

HRMS $\boldsymbol{m} / \boldsymbol{z}: 283.0540 \quad[\mathrm{M}+\mathrm{Na}]^{+}$(calcd for $\left[\mathrm{C}_{13} \mathrm{H}_{12} \mathrm{~N}_{2} \mathrm{O}_{3} \mathrm{~S}+\mathrm{Na}\right]^{+}:$299,0466).

IR $v_{\max }\left(\mathbf{c m}^{-1}\right.$; KBr pellets $): 1637(\mathrm{C}=\mathrm{O}) ; 3234$ $(\mathrm{N}-\mathrm{H})$.

(E)- $N^{\prime}$-(2-Hydroxy-3-nitrobenzylidene)thiophene2-carbohydrazide (3I)

Yield: $77 \%$; yellow solid; m.p. $215-216^{\circ} \mathrm{C}$.

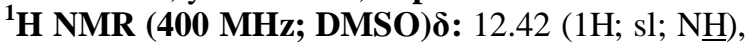
$12.00(1 \mathrm{H} ; \mathrm{sl}$; NH$), 8.70(1 \mathrm{H} ; \mathrm{s} ; \mathrm{H}-4$ '), $8.26(1 \mathrm{H} ; \mathrm{s}$; H-4'), 8.02-7.93 (5H; m; H-8', H-9' and H-10'), 7.76 $\left(1 \mathrm{H} ; \mathrm{dd} ; J_{H H}=5.9\right.$ and $3.6 \mathrm{~Hz} ; \mathrm{H}-10$ ') $7.25-7.24(1 \mathrm{H}$; $\mathrm{m} ; \mathrm{H}-5), \quad 7.16-7.13 \quad(2 \mathrm{H}, \mathrm{m}, \mathrm{H}-3$ and $\mathrm{H}-4)$. ${ }^{13}$ C NMR(100 MHz DMSO) $\boldsymbol{\delta}: 161.2(\mathrm{C}=\mathrm{O}), 157.7$ \& 151.0 (C-2), 151.5 \& 146.4 (C-4'), 139.8 \& 138.6 (C-6'), $137.6 \& 137.0$ (C-7'), $134.9 \& 132.8$ (C-10'), $132.7 \& 130.8$ (C-3), $129.7 \& 128.3$ (C-5), $128.0 \&$ 126.6 (C-4), 126.8 \& 123.7 (C-5'), 121.9 \& 120.1 (C-8'), $119.3 \& 118.6$ (C-9').

HRMS $\boldsymbol{m} / \boldsymbol{z}: \quad 314.0220 \quad[\mathrm{M}+\mathrm{Na}]^{+}$(calcd for $\left[\mathrm{C}_{12} \mathrm{H}_{9} \mathrm{~N}_{3} \mathrm{O}_{4} \mathrm{~S}+\mathrm{Na}\right]^{+}:$314.0212).

IR $v_{\max }\left(\mathbf{c m}^{-1} ; \mathrm{KBr}\right.$ pellets $): 1658(\mathrm{C}=\mathrm{O}) ; 3300$ $(\mathrm{N}-\mathrm{H})$

(E)-N'-(2-Hydroxy-5-nitrobenzylidene)thiophene2-carbohydrazide (3m)

Yield: $76 \%$; yellow solid; m.p. $195-196^{\circ} \mathrm{C}$.

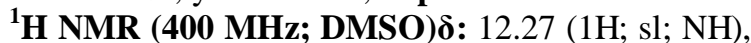
$8.72\left(1 \mathrm{H} ; \mathrm{d} ; J_{H H}=2.2 \mathrm{~Hz} ; \mathrm{H}-10\right.$ '), $8.60(1 \mathrm{H} ; \mathrm{s} ; \mathrm{H}-$ 4'), $8.18\left(1 \mathrm{H} ; \mathrm{dd} ; J_{H H}=8.9\right.$ and $2.2 \mathrm{~Hz} ; \mathrm{H}-8$ '), 8.05-7.92 $\left(2 \mathrm{H}\right.$; m; H-3 and H-5), $7.25\left(1 \mathrm{H} ; \mathrm{t} ; J_{H H}=\right.$ $4.1 \mathrm{~Hz} ; \mathrm{H}-4), 7.12\left(1 \mathrm{H} ; \mathrm{d} ; J_{H H}=9.0 \mathrm{~Hz} ; \mathrm{H}-7\right.$ '). ${ }^{13}$ C NMR(100 MHz DMSO) $\delta: 163.7 \&$ \& 162.5 $(\mathrm{C}=\mathrm{O}), 162.0 \& 161.4$ (C-6'), $160.1 \& 157.8$ (C-4'), $143.7 \& 140.0$ (C-2), $138.3 \& 137.6$ (C-9'), 135.07 132.4 (C-3), $129.5 \& 128.3$ (C-5), $126.8 \& 126.6$ (C-4), $125.1 \& 123.5$ (C-5'), $121.9 \& 121.3$ (C-10'), $120.2 \& 119.0$ (C-8'), 117.5 \& 117.1 (C-7'). 
HRMS $\boldsymbol{m} / \boldsymbol{z}: \quad 314.0230 \quad[\mathrm{M}+\mathrm{Na}]^{+} \quad$ (calcd for $\left.\left[\mathrm{C}_{12} \mathrm{H}_{9} \mathrm{~N}_{3} \mathrm{O}_{4} \mathrm{~S}+\mathrm{Na}\right]^{+}: 314,0212\right)$.

IR $v_{\max }\left(\mathbf{c m}^{-1} ; \mathrm{KBr}\right.$ pellets $): 1624(\mathrm{C}=\mathrm{O}) ; 3292$ (N-H).

\section{(E)-N'-(3-Chloro-4-hydroxy-}

benzylidene)thiophene-2-carbohydrazide (3n)

Yield: $69 \%$; light yellow solid; m.p. $212-213^{\circ} \mathrm{C}$.

${ }^{1}$ H NMR (400 MHz; DMSO)d: $11.80(1 \mathrm{H} ; \mathrm{sl} ; \mathrm{NH})$, 8.30 (1H; s; H-4'), 8.03-7.97 (1H; m; H-9'), 7.907.87 (1H; m; H-10'), 7.75-7.71 (1H; m; H-6'), $7.61-7.53(1 \mathrm{H} ; \mathrm{m} ; \mathrm{H}-3), 7.22\left(1 \mathrm{H} ; \mathrm{dd} ; J_{H H}=4.9\right.$ and 3.9 Hz; H-5), 7.10-7.05 (1 H; m; H-4). ${ }^{13} \mathbf{C}$ NMR(100 MHz DMSO) $\delta: 161.1 \quad(\mathrm{C}=\mathrm{O}), 154.8 \quad \& 146.6$ (C-8'), 154.7 (C-4'), 143.0 \& 138.3 (C-2), 134.9 (C3), 133.0 (C-5), 131.7 (C-4), $128.8 \& 128.7$ (C-5'), $128.4 \& 128.1$ (C-6'), $127.3 \& 126.6$ (C-10'), 126.5 \& 120.3 (C-7'), 117.0 \& 116.9 (C-9').

HRMS m/z: $302.9987 \quad[\mathrm{M}+\mathrm{Na}]^{+}$(calcd for $\left[\mathrm{C}_{12} \mathrm{H}_{9} \mathrm{ClN}_{2} \mathrm{O}_{2} \mathrm{~S}+\mathrm{Na}\right]^{+}:$302,9971).

IR $v_{\max }\left(\mathbf{c m}^{-1} ; \mathrm{KBr}\right.$ pellets $): 1624(\mathrm{C}=\mathrm{O}) ; 3255$ (N-H).

(E)- $N^{\prime}$-((5-Nitrothiophen-2-yl)methylene)furan-2carbohydrazide (4a)

Yield: 40\%; yellow solid; m.p. $209-210^{\circ} \mathrm{C}$.

${ }^{1}$ H NMR (400 MHz; DMSO) $\boldsymbol{\delta}: 12.24(1 \mathrm{H} ; \mathrm{s} ; \mathrm{NH})$, $8.66\left(1 \mathrm{H} ; \mathrm{s} ; \mathrm{H}-4\right.$ '), 8.13 (1H; d; $J_{H H}=4.3 \mathrm{~Hz} ; \mathrm{H}-8$ '), $7.99(1 \mathrm{H} ; \mathrm{s} ; \mathrm{H}-5), 7.57\left(1 \mathrm{H} ; \mathrm{d} ; J_{H H}=4.3 \mathrm{~Hz} ; \mathrm{H}^{-}{ }^{\prime}\right)$, 7.36 (1H; s; H-3), 6.74 (1H; s; H-4). ${ }^{13} \mathbf{C}$ NMR(100 MHz DMSO) $\boldsymbol{\delta}: 154.2$ (C=O), 150.8 (C-7'), 146.6 (C-4'), 146.4 (C-2), 146.2 (C-5), 141.1 (C-8'), 130.5 (C-9'), 129.6 (C-5'), 115.8 (C-4), 112.3 (C-3). HRMS $m / z$ : $288.0073 \quad[\mathrm{M}+\mathrm{Na}]^{+}$(calcd for $\left[\mathrm{C}_{10} \mathrm{H}_{7} \mathrm{~N}_{3} \mathrm{O}_{4} \mathrm{~S}+\mathrm{Na}\right]^{+}:$288.0055).

IR $\mathbf{v}_{\max }\left(\mathbf{c m}^{-1} ; \mathrm{KBr}\right.$ pellets $): 1659(\mathrm{C}=\mathrm{O}) ; 3155$ (N-H).

(E)- $N^{\prime}$-((5-Nitrofuran-2-yl)methylene)furan-2carbohydrazide (4b)

Yield: 84\%; yellow solid; m.p. $167-169^{\circ} \mathrm{C}$;

${ }^{1}$ H NMR (400 MHz; DMSO) $\delta$ : $12.28(1 \mathrm{H} ; \mathrm{s} ; \mathrm{NH})$, 8.40 (1H; s; H-4'), 8.00 (1H; s; H-5), $7.80(1 \mathrm{H}$; d; $\left.J_{H H}=3.9 \mathrm{~Hz} ; \mathrm{H}-8^{\prime}\right), 7.37(1 \mathrm{H} ; \mathrm{sl} ; \mathrm{H}-3), 7.28(1 \mathrm{H} ; \mathrm{d}$; $\left.J_{H H}=3.9 \mathrm{~Hz} ; \mathrm{H}_{-9}{ }^{\prime}\right), 6.74\left(1 \mathrm{H} ; \mathrm{dd} ; J_{H H}=3.3\right.$ and 1.5 $\mathrm{Hz} ; \mathrm{H}-4) .{ }^{13} \mathbf{C}$ NMR(100 MHz DMSO) $\boldsymbol{\delta}: 154.2$ $(\mathrm{C}=\mathrm{O}), 151.8$ (C-7), 151.6 (C-4'), 146.3 (C-2), 146.0 (C-5), 135.4 (C-8), 115.2 (C-9'), 114.6 (C-4), 112.2 (C-3).

HRMS $m / z: 272.0292 \quad[\mathrm{M}+\mathrm{Na}]^{+}$(calcd for $\left[\mathrm{C}_{10} \mathrm{H}_{7} \mathrm{~N}_{3} \mathrm{O}_{5}+\mathrm{Na}\right]^{+}:$272,0283).

IR $v_{\max }\left(\mathbf{c m}^{-1} ; \mathrm{KBr}\right.$ pellets $): 1666(\mathrm{C}=\mathrm{O}), 3124$ $(\mathrm{N}-\mathrm{H})$.

\section{(E)- $N^{\prime}$-(Pyridin-2-ylmethylene)furan-2-}

carbohydrazide (4c)

Yield: $32 \%$; brown solid; m.p. $149-150^{\circ} \mathrm{C}$.

${ }^{1}$ H NMR (400 MHz; DMSO) $\delta$ : 12.09 (1H; s; NH), $8.63\left(1 \mathrm{H} ; \mathrm{d} ; J_{H H}=4.6 \mathrm{~Hz}\right.$; H-7'), $8.49(1 \mathrm{H} ; \mathrm{s} ; \mathrm{H}-4$ '), $7.97\left(2 \mathrm{H} ; \mathrm{d} ; J_{H H}=7.9 \mathrm{~Hz} ; \mathrm{H}-8\right.$ ' and H-9'), 7.91-7.87 (1H; m; H-5), 7.44-7.41 (1H; m; H-10'), 7.35 (1H; s; H-3), 6.74-6.73 (1H; m; H-4). ${ }^{13} \mathbf{C}$ NMR(100 MHz DMSO) $\boldsymbol{\delta}: 153.7(\mathrm{C}=\mathrm{O}), 152.6\left(\mathrm{C}-5^{\prime}\right), 148.9$ (C-7'), 147.5 (C-4'), 145.8 (C-2), 145.1 (C-5), 136.3 (C-9'),
123.8 (C-10'), 119.4 (C-8'), 114.9 (C-4), 111.6 (C-3).

HRMS $\boldsymbol{m} / \boldsymbol{z}: 238.0601 \quad[\mathrm{M}+\mathrm{Na}]^{+}$(calcd for $\left[\mathrm{C}_{11} \mathrm{H}_{9} \mathrm{~N}_{3} \mathrm{O}_{2}+\mathrm{Na}\right]^{+}$: 238.0593).

IR $v_{\max }\left(\mathbf{c m}^{-1} ; \mathrm{KBr}\right.$ pellets $): 1655(\mathrm{C}=\mathrm{O}) ; 3133$ $(\mathrm{N}-\mathrm{H})$.

(E)- $N^{\prime}$-(2-Hydroxybenzylidene)furan-2-

carbohydrazide (4d)

Yield: $66 \%$; light yellow solid; m.p. $146-147^{\circ} \mathrm{C}$.

${ }^{1}$ H NMR (400 MHz; DMSO) $\delta$ : $12.11(1 \mathrm{H} ; \mathrm{s} ; \mathrm{OH})$, 11.14 (1H; sl; NH), 8.64 (1H; s; H-4'), 7.96 (1H; d; $J_{H H}=1.0 \mathrm{HZ}$; H-5), $7.53\left(1 \mathrm{H} ; \mathrm{d} ; J_{H H}=7.4 \mathrm{~Hz} ; \mathrm{H}-3\right)$, $7.30\left(2 \mathrm{H} ; \mathrm{d} ; J_{H H}=7.8 \mathrm{~Hz} ; \mathrm{H}-8\right.$ ' and $\left.\mathrm{H}-10 '\right), 6.93$ $\left(2 \mathrm{H} ; \mathrm{d} ; J_{H H}=7.9 \mathrm{~Hz} ; \mathrm{H}-7\right.$ ' and H-9'), $6.72(1 \mathrm{H}$; dd; $J_{H H}=3.4$ and H-4). ${ }^{13} \mathbf{C}$ NMR(100 MHz DMSO) $\boldsymbol{\delta}$ : 157.4 (C=O), 154.0 (C-6'), 148.3 (C-4'), 146.3 (C2), 146.1 (C-5), 131.4 (C-10'), 129.4 (C-9'), 119.4 (C-8'), 118.8 (C-5'), 116.4 (C-7'), 115.3 (C-4), $112.2(\mathrm{C}-3)$.

HRMS $m / z: 253.0567 \quad[\mathrm{M}+\mathrm{Na}]^{+}$(calcd for $\left[\mathrm{C}_{12} \mathrm{H}_{10} \mathrm{~N}_{2} \mathrm{O}_{3}+\mathrm{Na}\right]^{+}$: 253.0589).

IR $v_{\max }\left(\mathbf{c m}^{-1} ; \mathrm{KBr}\right.$ pellets $): 1621(\mathrm{C}=\mathrm{O}) ; 3130$ (N-H).

\section{(E)-N'-(4-Hydroxybenzylidene)furan-2-}

carbohydrazide (4e)

Yield: 45\%; light yellow solid; m.p. $239-240^{\circ} \mathrm{C}$.

${ }^{1}$ H NMR (400 MHz; DMSO)反: $11.62(1 \mathrm{H} ; \mathrm{sl} ; \mathrm{NH})$, 8.35 (1H; s; H-4'), 7.69 (1H; d; $J_{H H}=8.6 \mathrm{HZ}$; H-9'), $7.54\left(2 \mathrm{H} ; \mathrm{d} ; J_{H H}=8.6 \mathrm{~Hz} ; \mathrm{H}-7\right.$ ' and H-10'), 7.27 $\left(1 \mathrm{H} ; \mathrm{d} ; J_{H H}=2.4 \mathrm{~Hz} ; \mathrm{H}-6\right.$ '), 6.83-6.88 $(3 \mathrm{H} ; \mathrm{m} ; \mathrm{H}-3$, $\mathrm{H}-4$ and H-5). ${ }^{13} \mathbf{C}$ NMR(100 MHz DMSO) $\delta: 160.2$ $(\mathrm{C}=\mathrm{O}), 153.9$ (C-8'), $148.1 \quad(\mathrm{C}-4$ '), 146.7 (C-2), 145.5 (C-5), 128.8 (C-5'), 125.1 (C-10'), 125.0 (C6'), 115.6 (C-9'), 114.4 (C-7'), 111.9 (C-4), 111.5 (C-3).

HRMS $m / z: 253.0570 \quad[\mathrm{M}+\mathrm{Na}]^{+}$(calcd for $\left[\mathrm{C}_{12} \mathrm{H}_{10} \mathrm{~N}_{2} \mathrm{O}_{3}+\mathrm{Na}\right]^{+}:$253.05891).

IR $\mathbf{v}_{\max }\left(\mathbf{c m}^{-1} ; \mathrm{KBr}\right.$ pellets): $1587(\mathrm{C}=\mathrm{O}) ; 3175$ (N-H).

(E)- $N^{\prime}$-(2,3-Dihydroxybenzylidene)furan-2carbohydrazide (4f)

Yield: $56 \%$; light yellow solid; m.p. $121-122^{\circ} \mathrm{C}$.

${ }^{1}$ H NMR (400 MHz; DMSO) $\delta: 12.14(1 \mathrm{H} ; \mathrm{sl}$; NH), 8.66 (1H; s; H-4'), 7.97 (1H; s; H-5),

7.32-7.28 (1H; m; H-9'), $7.14\left(1 \mathrm{H} ; \mathrm{dd} ; J_{H H}=7.8\right.$ and $1.4 \mathrm{~Hz}$; H-8'), $6.95\left(1 \mathrm{H}\right.$; dd; $J_{H H}=7.8$ and $1.3 \mathrm{~Hz}$; H-10'), 6.88-6.85 (1H; m; H-3), 6.73-6.71 (1H; m; $\mathrm{H}-4) .{ }^{13}$ C NMR(100 MHz DMSO) $\delta: 153.8(\mathrm{C}=\mathrm{O})$, 148.8 (C-4'), 146.9 (C-2), 146.0 (C-5), 145.9 (C-6'), 121.2 (C-7'), 120.5 (C-5'), 119.8 (C-9'), 118.3 (C8'), 117.3 (C-10'), 113.7 (C-4), 112.1 (C-3).

HRMS $\boldsymbol{m} / \boldsymbol{z}: \quad 269.0540 \quad[\mathrm{M}+\mathrm{Na}]^{+}$(calcd for $\left[\mathrm{C}_{12} \mathrm{H}_{10} \mathrm{~N}_{2} \mathrm{O}_{4}+\mathrm{Na}\right]^{+}:$269.0538).

IR $v_{\max }\left(\mathbf{c m}^{-1}\right.$; KBr pellets $): 1645(\mathrm{C}=\mathrm{O}) ; 3120$ $(\mathrm{N}-\mathrm{H})$.

(E)- $N^{\prime}$-(2-Hydroxy-4-methylbenzylidene)furan-2carbohydrazide (4g)

Yield: 50\%; light yellow solid; m.p. $203-204^{\circ} \mathrm{C}$.

${ }^{1}$ H NMR (400 MHz; DMSO)d: $12.06(1 \mathrm{H} ; \mathrm{sl} ; \mathrm{NH})$, $8.93\left(1 \mathrm{H} ; \mathrm{s} ; \mathrm{H}-4\right.$ '), $7.96\left(1 \mathrm{H}\right.$; dd; $J_{H H}=1.6$ and 0.6 
Hz; H-7'), 7.30 (1H; d; $\left.J_{H H}=3.1 \mathrm{~Hz} ; \mathrm{H}-5\right), 6.81-6.79$ (1H; m; H-3), 6.76-6.74 (2H; m; H-9' and H-10'), 6.72-6.71 (1H; m; H-4), $2.28 \quad\left(3 \mathrm{H} ; \quad \mathrm{s} ; \mathrm{CH}_{3}\right)$. ${ }^{13}$ C NMR(100 MHz DMSO) $\boldsymbol{\delta}: 153.8(\mathrm{C}=\mathrm{O}), 148.3$ (C-4'), 146.2 (C-2), 145.9 (C-5), 143.7 (C-6'), 130.8 (C-8'), 129.3 (C-10'), 120.3 (C-5'), 116.6 (C-9'), 115.5 (C-7'), 115.0 (C-4), 112.0 (C-3), $21.1\left(\underline{\mathrm{CH}}_{3}\right)$. HRMS $\boldsymbol{m} / \boldsymbol{z}: 267.0746[\mathrm{M}+\mathrm{Na}]^{+}$(calcd for $\left[\mathrm{C}_{13} \mathrm{H}_{12} \mathrm{~N}_{2} \mathrm{O}_{3}+\mathrm{Na}\right]^{+}$: 267.0746).

IR $v_{\max }\left(\mathbf{c m}^{-1}\right.$; KBr pellets): $1607(\mathrm{C}=\mathrm{O}) ; 2919$ $(\mathrm{N}-\mathrm{H})$.

(E)- $N^{\prime}$-(2-Hydroxy-5-methylbenzylidene)furan-2carbohydrazide (4h)

Yield: 60\%; light yellow solid; m.p. $93-94^{\circ} \mathrm{C}$.

${ }^{1}$ H NMR (400 MHz; DMSO)d: 12.09 (1H; sl; NH), $10.88(1 \mathrm{H} ; \mathrm{s} ; \mathrm{OH}) 8.60(1 \mathrm{H} ; \mathrm{s} ; \mathrm{H}-4$ '), $7.96(1 \mathrm{H}$; dd; $J_{H H}=1.7$ and $0.7 \mathrm{~Hz} ; \mathrm{H}-10$ '), $7.32\left(2 \mathrm{H} ; \mathrm{d} ; J_{H H}=3.2\right.$ $\mathrm{Hz}$; $\mathrm{H}-4$ and $\mathrm{H}-5), 7.10\left(1 \mathrm{H}\right.$; dd; $J_{H H}=8.3$ and 1.7 $\mathrm{Hz}$; H-8'), 6.82 (1H; d; $\left.J_{H H}=8.3 \mathrm{~Hz} ; \mathrm{H}^{\prime} 7^{\prime}\right), 6.72$ $\left(1 \mathrm{H} ; \mathrm{dd} ; J_{H H}=3.5\right.$ and $\left.1.7 ; \mathrm{H}-3\right), 2.25\left(3 \mathrm{H} ; \mathrm{s} ; \mathrm{CH}_{3}\right)$. ${ }^{13}$ C NMR(100 MHz DMSO) $\delta$ : $155.1(\mathrm{C}=\mathrm{O}), 153.8$ (C-4'), 147.95 (C-2), 146.2 (C-5), 145.9 (C-6'), 132.0 (C-8'), 129.0 (C-9'), 127.8 (C-10'), 118.3 (C-5'), 116.1 (C-7'), 115.1 (C-4), 112.1 (C-3), 19.8 $\left(\mathrm{CH}_{3}\right)$.

HRMS $\boldsymbol{m} / \boldsymbol{z}: 267.0758 \quad[\mathrm{M}+\mathrm{Na}]^{+}$(calcd for $\left[\mathrm{C}_{13} \mathrm{H}_{12} \mathrm{~N}_{2} \mathrm{O}_{3}+\mathrm{Na}\right]^{+}:$267,0746).

IR $v_{\max }\left(\mathbf{c m}^{-1} ; \mathrm{KBr}\right.$ pellets $): 1628(\mathrm{C}=\mathrm{O}) ; 3011$ $(\mathrm{N}-\mathrm{H})$.

(E)- $N^{\prime}$-(2-Hydroxy-3-methoxybenzylidene)furan2-carbohydrazide (4i)

Yield: 57\%; yellow solid; m.p. $122-123^{\circ} \mathrm{C}$.

${ }^{1}$ H NMR (400 MHz; DMSO) $\mathbf{~ : ~} 12.10(1 \mathrm{H} ; \mathrm{s} ; \mathrm{NH})$, $10.80(1 \mathrm{H} ; \mathrm{s} ; \mathrm{OH}), 8.67$ (1H; s; H-4'), $7.96(1 \mathrm{H}$; dd; $J_{H H}=1.7$ and $\left.0.7 \mathrm{~Hz} ; \mathrm{H}-5\right), 7.32\left(1 \mathrm{H} ; \mathrm{d} ; J_{H H}=2.9\right.$ $\mathrm{Hz}$; H-3), $7.15\left(1 \mathrm{H}\right.$; d; $J_{H H}=7.6 \mathrm{~Hz} ; \mathrm{H}-8$ '), $7.04(1 \mathrm{H}$; $\mathrm{dd} ; J_{H H}=7.9$ and $0.9 \mathrm{~Hz} ; \mathrm{H}-10$ '), $6.86\left(1 \mathrm{H} ; \mathrm{t} ; J_{H H}=\right.$ $7.9 \mathrm{~Hz} ; \mathrm{H}-9$ '), $6.71\left(1 \mathrm{H}\right.$; dd; $J_{H H}=3.5$ and $1.7 \mathrm{~Hz}$; $\mathrm{H}-4), 3.82\left(3 \mathrm{H} ; \mathrm{s} ; \mathrm{CH}_{3}\right) .{ }^{13} \mathbf{C} \quad \mathbf{N M R}(\mathbf{1 0 0} \mathbf{M H z}$ DMSO) $\delta: 154.0(\mathrm{C}=\mathrm{O}), 148.0(\mathrm{C}-6$ ') 147.9 (C-6'), 147.1 (C-7'), 146.3 (C-2), 145.6 (C-4'), 144.9 (C-5), 120.6 (C-10'), 119.1 (C-5'), 115.2 (C-9'), 113.8 (C-3 or C-8'), 112.2 (C-3 or C-8'), 111,5 (C-4), 55.8 $\left(\mathrm{CH}_{3}\right)$.

HRMS $\boldsymbol{m} / \boldsymbol{z}: 283.0700 \quad[\mathrm{M}+\mathrm{Na}]^{+}$(calcd for $\left[\mathrm{C}_{13} \mathrm{H}_{12} \mathrm{~N}_{2} \mathrm{O}_{4}+\mathrm{Na}\right]^{+}$: 283.0695).

IR $v_{\max }\left(\mathbf{c m}^{-1} ; \mathrm{KBr}\right.$ pellets $): 1651(\mathrm{C}=\mathrm{O}) ; 3131$ $(\mathrm{N}-\mathrm{H})$.

(E)- $N^{\prime}$-(2-Hydroxy-4-methoxybenzylidene)furan2-carbohydrazide (4j)

Yield: $62 \%$; yellow solid; m.p. $152-153^{\circ} \mathrm{C}$.

${ }^{1}$ H NMR (400 MHz; DMSO)d: $12.00(1 \mathrm{H} ; \mathrm{s} ; \mathrm{OH})$, $11.47(1 \mathrm{H} ; \mathrm{sl} ; \mathrm{NH}), 8.55(1 \mathrm{H} ; \mathrm{s} ; \mathrm{H}-4$ '), $7.95(1 \mathrm{H} ; \mathrm{d}$; $\left.J_{H H}=1.0 \mathrm{HZ} ; \mathrm{H}-5\right), 7.41\left(1 \mathrm{H} ; \mathrm{d} ; J_{H H}=8.5 \mathrm{~Hz}\right.$; H-10'), $7.29\left(1 \mathrm{H} ; \quad \mathrm{d} ; \quad J_{H H}=3.1 \mathrm{~Hz} ; \mathrm{H}-3\right)$, $6.71\left(1 \mathrm{H} ; \mathrm{dd} ; J_{H H}=3.5\right.$ and $\left.1.7 \mathrm{~Hz} ; \mathrm{H}-4\right), 6.54-6.49$ $\left(2 \mathrm{H} ; \mathrm{m} ; \mathrm{H}^{-}{ }^{\prime}\right.$ and $\left.\mathrm{H}-9^{\prime}\right), 3.78\left(3 \mathrm{H} ; \mathrm{s} ; \mathrm{CH}_{3}\right)$. ${ }^{13}$ C NMR(100 MHz DMSO) $\delta: 162.0(\mathrm{C}=\mathrm{O}), 159.2$ (C-8'), 153.7 (C-6'), 148.6 (C-4'), 146.2 (C-2),
145.5 (C-5), 130. (C-10’), 114.9 (C-5'), 112.0 (C-4), 111,7 (C-3), 106.4 (C-9'), 101.0 (C-7'), $55.2\left(\right.$ CH$\left._{3}\right)$. HRMS $\boldsymbol{m} / \boldsymbol{z}: 283.0696[\mathrm{M}+\mathrm{Na}]^{+}$(calcd for $\left[\mathrm{C}_{13} \mathrm{H}_{12} \mathrm{~N}_{2} \mathrm{O}_{4}+\mathrm{Na}\right]^{+}$: 283.0695).

IR $v_{\max }\left(\mathbf{c m}^{-1}\right.$; KBr pellets $): 1644(\mathrm{C}=\mathrm{O}) ; 3165$ $(\mathrm{N}-\mathrm{H})$.

\section{(E)- $N^{\prime}$-(3-Chloro-4-hydroxy-}

benzylidene)thiophene-2-carbohydrazide (4k)

Yield: $42 \%$; yellow solid; m.p. $202-203^{\circ} \mathrm{C}$.

${ }^{1}$ H NMR (400 MHz; DMSO) $\delta$ : $11.76(1 \mathrm{H} ; \mathrm{s} ; \mathrm{NH})$, $10.70(1 \mathrm{H} ; \mathrm{s} ; \mathrm{OH}), 8.30(1 \mathrm{H} ; \mathrm{s} ; \mathrm{H}-4$ '), $7.79(1 \mathrm{H} ; \mathrm{d}$; $J_{H H}=1.0 \mathrm{HZ}$; H-5), $7.51\left(1 \mathrm{H} ; \mathrm{dd} ; J_{H H}=8.4\right.$ and 1.8 $\mathrm{Hz}$; H-10'), 7.28 (1H; s; H-6'), $7.07\left(1 \mathrm{H} ; \mathrm{d} ; J_{H H}=\right.$ $3.2 \mathrm{~Hz}$; H-3), 7.04 (1H; d; $J_{H H}=8.4 \mathrm{~Hz} ; \mathrm{H}^{\prime} 9$ '), 6.59 $\left(1 \mathrm{H} ; \mathrm{dd} ; J_{H H}=3.5\right.$ and $\left.1.7 \mathrm{~Hz} ; \mathrm{H}-4\right) .{ }^{13} \mathbf{C}$ NMR(100 MHz DMSO) $\delta$ : $157.9(\mathrm{C}=\mathrm{O}), 154.1$ (C-8'), 147.0 (C-4'), 146.7 (C-2), 145.7 (C-5), 128.4 (C-5'), 127.2 (C-6'), 126.6 (C-10'), 120.3 (C-7'), 114.8 (C-9'), 112.0 (C-4), 111.5 (C-3).

HRMS m/z: $303.0204 \quad[\mathrm{M}+\mathrm{K}]^{+} \quad($ calcd for $\left[\mathrm{C}_{12} \mathrm{H}_{9} \mathrm{ClN}_{2} \mathrm{O}_{3}+\mathrm{K}\right]^{+}:$302.9939).

IR $v_{\max }\left(\mathbf{c m}^{-1}\right.$; KBr pellets $): 1640(\mathrm{C}=\mathrm{O}) ; 3127$ $(\mathrm{N}-\mathrm{H})$.

\section{General Synthesis of 5 and 6.}

The appropriate derivative $2^{11}$ or 3 ( $0.2 \mathrm{~g}, 1.0$ equiv.) was suspended in acetone $(5.0 \mathrm{~mL})$ and potassium carbonate (4.0 equiv.) was added. The reaction mixture was stirred at room temperature for 30 minutes and methyl iodide (4.0 equiv.) was added. The temperature of the reaction mixture was increased to $40^{\circ} \mathrm{C}$ over $2-24$ hours. The reaction mixture was rotary evaporated to leave a residue which was dissolved in water $(20.0 \mathrm{~mL})$ and extracted with ethyl acetate $(3 \times 10.0 \mathrm{~mL})$. The organic phases were combined, dried with anhydrous magnesium sulfate, filtered and then evaporated at reduced pressure. The derivatives 5 and $\mathbf{6}$ were obtained as solids in $65-89 \%$ yields.

(E)-N-Methyl- $N^{\prime}$-((5-nitrothiophen-2-

yl)methylene)thiophene-2-carbohydrazide (5a):

Yield: $89 \%$; yellow solid; m.p. $180-181^{\circ} \mathrm{C}$.

${ }^{1}$ H NMR (400 MHz; DMSO) $\boldsymbol{~}: 8.38$ (1H; s; H-4'), $8.15\left(1 \mathrm{H} ; \mathrm{d} ; J_{H H}=4.3 \mathrm{~Hz} ; \mathrm{H}-8\right.$ '), $8.07\left(1 \mathrm{H} ; \mathrm{dd} ; J_{H H}=\right.$ 5.0 and $1.3 \mathrm{~Hz} ; \mathrm{H}-5), 8.04\left(1 \mathrm{H}\right.$; dd; $J_{H H}=3.8$ and 1.3 $\mathrm{Hz}$; H-3), 7.57 (1H; d; $J_{H H}=4.3 \mathrm{~Hz} \mathrm{H}-9$ '), $7.22(1 \mathrm{H}$; $\mathrm{dd} ; J_{H H}=5.0$ and $\left.3.8 \mathrm{~Hz} ; \mathrm{H}-4\right), 3.50\left(3 \mathrm{H} ; \mathrm{s} ; \mathrm{CH}_{3}\right)$. ${ }^{13}$ C NMR(100 MHz DMSO) $\boldsymbol{\delta}: 161.1(\mathrm{C}=\mathrm{O}), 151.0$ (C-4'), 146.4 (C-7'), 136.3 (C-8' and C-9'), 135.4 (C-5'), 131.9 (C-2), 130.6 (C-3), 130.1 (C-4), 126.6 (C-5), $29.4\left(\mathrm{CH}_{3}\right)$.

HRMS $\boldsymbol{m} / \boldsymbol{z}: 317.9990 \quad[\mathrm{M}+\mathrm{Na}]^{+}$(calcd for $\left[\mathrm{C}_{11} \mathrm{H}_{9} \mathrm{~N}_{3} \mathrm{O}_{3} \mathrm{~S}_{2}+\mathrm{Na}\right]^{+}$: 317,9983).

IR $v_{\max }\left(\mathbf{c m}^{-1}\right.$; KBr pellets): $1637(\mathrm{C}=\mathrm{O}) ; 1168$ $(\mathrm{C}-\mathrm{N}) .(\boldsymbol{E})-\mathrm{N}$-Methyl- $N^{\prime}$-((5-nitrofuran-2-

yl)methylene)thiophene-2-carbohydrazide (5b):

Yield: $76 \%$; dark yellow solid; m.p. $165-166^{\circ} \mathrm{C}$.

${ }^{1}$ H NMR (400 MHz; DMSO) $\boldsymbol{\delta}$ : 8.09 (2H; m; H-4' and $\mathrm{H}-5), 7.99\left(1 \mathrm{H}\right.$; d; $\left.J_{H H}=4.4 \mathrm{~Hz} ; \mathrm{H}-3\right), 7.82(1 \mathrm{H}$; $\mathrm{d} ; J_{H H}=3.8 \mathrm{~Hz} ; \mathrm{H}-8$ '), $7.24\left(1 \mathrm{H} ; \mathrm{d} ; J_{H H}=3.9 \mathrm{~Hz} ; \mathrm{H}-\right.$ 9'), $7.20\left(1 \mathrm{H} ; \quad \mathrm{t} ; \quad J_{H H}=4.5 \mathrm{~Hz} \quad \mathrm{H}-4\right)$, 
3.49 (3H; s; $\left.\mathrm{CH}_{3}\right) .{ }^{\mathbf{1 3}} \mathbf{C}$ NMR(100 MHz DMSO) $\boldsymbol{\delta}:$ $161.4(\mathrm{C}=\mathrm{O}), 152.4$ (C-4'), 151.7 (C-5'), 136.2 (C-2 and C-3), 132.4 (C-7'), 129.2 (C-4), 126.7 (C-5), 114.8 (C-9'), 114.3 (C-8'), $29.0\left(\mathrm{CH}_{3}\right)$.

HRMS $m / z: 302.0211 \quad[\mathrm{M}+\mathrm{Na}]^{+}$(calcd for $\left.\left[\mathrm{C}_{11} \mathrm{H}_{9} \mathrm{~N}_{3} \mathrm{O}_{4} \mathrm{~S}+\mathrm{Na}\right]^{+}: 302.0212\right)$.

IR $v_{\max }\left(\mathbf{c m}^{-1}\right.$; KBr pellets $): 1656(\mathrm{C}=\mathrm{O}) ; 1190$ (C-N).

(E)-N-Methyl- $N$ '-(pyridin-2-

ylmethylene)thiophene-2-carbohydrazide (5c):

Yield: $88 \%$; brown solid; m.p. $90-91{ }^{\circ} \mathrm{C}$.

${ }^{1}$ H NMR (400 MHz; DMSO)d: $8.68\left(1 \mathrm{H} ; \mathrm{d} ; J_{H H}=\right.$ $4.5 \mathrm{~Hz}$; H-7'), 8.18 (1H; d; $J_{H H}=7.9 \mathrm{~Hz}$; H-9'), 8.09 $\left(1 \mathrm{H} ; \mathrm{dd} ; J_{H H}=3.8\right.$ and $\left.1.2 \mathrm{~Hz} ; \mathrm{H}-5\right), 8.05(1 \mathrm{H} ; \mathrm{s}$; H-4'), 8.00-7.94 (2H; m; H-3 and H-10'), 7.45 (1H; $\mathrm{dd} ; J_{H H}=7.2$ and $5.6 \mathrm{~Hz} ; \mathrm{H}-8$ '), $7.22\left(1 \mathrm{H} ; \mathrm{t} ; J_{H H}=\right.$ 4.8; H-4) 3.55 (3H; s; $\left.\mathrm{CH}_{3}\right) .{ }^{13} \mathbf{C}$ NMR(100 MHz DMSO) $\delta: 161.4(\mathrm{C}=\mathrm{O}), 153.2\left(\mathrm{C}-4^{\prime}\right), 149.6\left(\mathrm{C}-7^{\prime}\right)$, 142.1 (C-5'), 137.0 (C-9'), 136.0 (C-2), 135.6 (C-3), 132.4 (C-4), 126.6 (C-5), 124.1 (C-10'), 120.8 (C-8'), $29.4\left(\mathrm{CH}_{3}\right)$.

HRMS $m / z: 268.0529 \quad[\mathrm{M}+\mathrm{Na}]^{+} \quad$ (calcd for $\left[\mathrm{C}_{12} \mathrm{H}_{11} \mathrm{~N}_{3} \mathrm{OS}+\mathrm{Na}\right]^{+}:$268,0521).

IR $v_{\max }\left(\mathbf{c m}^{-1} ; \mathrm{KBr}\right.$ pellets $): 1641(\mathrm{C}=\mathrm{O}) ; 1166$ (C-N).

(E)-N-Methyl- $N^{\prime}-((5-n i t r o t h i o p h e n-2-y l)-$ methylene)-2-(thiophen-2-yl)acetohydrazide (6a): Yield: $86 \%$; brown solid; m.p. $127-128^{\circ} \mathrm{C}$.

${ }^{1}$ H NMR (400 MHz; DMSO) $\boldsymbol{0}$ : 8.24 (1H; s; H-5'), $8.14\left(1 \mathrm{H} ; \mathrm{d} ; J_{H H}=4.3 \mathrm{~Hz}\right.$; H-9'), $7.51\left(1 \mathrm{H} ; \mathrm{d} ; J_{H H}=\right.$ $4.4 \mathrm{~Hz} ; \mathrm{H}-10$ '), $7.38\left(1 \mathrm{H}\right.$; dd; $J_{H H}=5.0$ and $1.3 \mathrm{~Hz}$; $\mathrm{H}-5)$, 6.98-6.96 (2H; m; H-3 and $\mathrm{H}-4), 4.31(2 \mathrm{H}$; s; H-1'), $3.32\left(3 \mathrm{H} ; \mathrm{s} ; \mathrm{C}_{3}\right) .{ }^{13} \mathbf{C} \mathbf{N M R}(\mathbf{1 0 0} \mathbf{M H z}$ DMSO) $\delta: 171.6(\mathrm{C}=\mathrm{O}), 150.4\left(\mathrm{C}-5^{\prime}\right), 147.1$ (C-2), 136.4 (C-6'), 134.3 (C-8'), 130.6 (C-5), 128.9 (C-3), 126.7 (C-4), 126.5 (C-9'), 125.3 (C-10'), 33.9 (C-1'), $28.5\left(\mathrm{CH}_{3}\right)$.

HRMS $\boldsymbol{m} / \mathbf{z}: 332.0165 \quad[\mathrm{M}+\mathrm{K}]^{+} \quad($ calcd for $\left[\mathrm{C}_{12} \mathrm{H}_{11} \mathrm{~N}_{3} \mathrm{O}_{4} \mathrm{~S}+\mathrm{K}\right]^{+}:$332,0107).

IR $v_{\max }\left(\mathbf{c m}^{-1} ; \mathrm{KBr}\right.$ pellets $): 1671(\mathrm{C}=\mathrm{O}) ; 1138$ (C-N).

(E)- $N$-Methyl- $N^{\prime}-((5-n i t r o f u r a n-2-y l) m e t h y l e n e)-$ 2-(thiophen-2-yl)acetohydrazide (6b):

Yield: 65\%; brown solid; m.p. $103-104^{\circ} \mathrm{C}$.

${ }^{1}$ H NMR (400 MHz; DMSO) $\boldsymbol{\delta}$ : 8.31 (1H; s; H-5'), 7.82-7.81 (3H; m; H-3; H-4 and H-5), 7.38-7.36 (4H; m; H-9' and H-10'), 7.25-7.24 (3H; m; H-3; H-4 and H-5), 4.34 (4H; s; H-1'). ${ }^{13} \mathbf{C}$ NMR(100 MHz DMSO) $\delta$ : $171.0(\mathrm{C}=\mathrm{O}), 153.6 \& 152.3\left(\mathrm{C}-5^{\prime}\right)$, $151.6 \& 143.4$ (C-6'), $136.5 \& 129.1$ (C-8'), 129.0 (C-2), 127.0 (C-3), 126.6 (C-5), 125.3 (C-4), 114.8 \& 114.7 (C-10'), 113.8 \& 113.7 (C-9'), 34.0 (C-1'), $28.7 \& 28.4\left(\mathrm{CH}_{3}\right)$.

HRMS m/z: $316.0394 \quad[\mathrm{M}+\mathrm{K}]^{+}$(calcd for $\left.\left[\mathrm{C}_{11} \mathrm{H}_{9} \mathrm{~N}_{3} \mathrm{O}_{4} \mathrm{~S}+\mathrm{K}\right]^{+}: 317,9951\right)$.

IR $v_{\max }\left(\mathbf{c m}^{-1} ; \mathrm{KBr}\right.$ pellets $): 1683(\mathrm{C}=\mathrm{O}) ; 1255$ (C-N).

(E)-N-Methyl- $N^{\prime}$-(pyridin-2-ylmethylene)-2(thiophen-2-yl)acetohydrazide (6c):

Yield: $67 \%$; brown solid; m.p. $81-82^{\circ} \mathrm{C}$.
${ }^{1}$ H NMR (400 MHz; DMSO) $\mathbf{0}: 8.62\left(1 \mathrm{H} ; \mathrm{d} ; J_{H H}=\right.$ $4.3 \mathrm{~Hz}$; H-8'), 8.06 (1H; d; $J_{H H}=7.9 \mathrm{~Hz} ; \mathrm{H}-10$ '), $7.89\left(1 \mathrm{H} ; \mathrm{d} ; J_{H H}=1.5 \mathrm{~Hz}\right.$; H-9'), $7.89(1 \mathrm{H} ; \mathrm{s} ; \mathrm{H}-5$ '), $7.41\left(1 \mathrm{H} ; \mathrm{d} ; J_{H H}=6.6\right.$ and $1.5 \mathrm{~Hz} ; \mathrm{H}-11$ '), $7.35(1 \mathrm{H}$; $\mathrm{dd} ; J_{H H}=5.1$ and $\left.1.1 \mathrm{~Hz} ; \mathrm{H}-5\right), 6.99\left(1 \mathrm{H} ; \mathrm{d} ; J_{H H}=\right.$ $2.6 \mathrm{~Hz}$; H-3), 6.95-6.93 (1H; m; H-4), 4.39 (2H; s; H-1'), 3.35 (3H; s; $\left.\mathrm{CH}_{3}\right) .{ }^{\mathbf{1 3}} \mathbf{C} \mathbf{N M R}(\mathbf{1 0 0} \mathbf{M H z}$ DMSO) $\delta: 171.1(\mathrm{C}=\mathrm{O}), 153.4\left(\mathrm{C}-5^{\prime}\right), 149.5\left(\mathrm{C}-8^{\prime}\right)$, 137.0 (C-10'), 136.9 (C-2), 126.8 (C-3), 126.6 (C-4), 125.3 (C-5), 124.2 (C-11'), 120.0 (C-9'), 34.3 (C-1'), $28.1\left(\underline{\mathrm{CH}}_{3}\right)$.

HRMS $m / z: 282.0685 \quad[\mathrm{M}+\mathrm{Na}]^{+}$(calcd for $\left[\mathrm{C}_{13} \mathrm{H}_{13} \mathrm{~N}_{3} \mathrm{OS}+\mathrm{Na}\right]^{+}:$282,0677).

IR $v_{\max }\left(\mathbf{c m}^{-1} ; \mathbf{K B r}\right.$ pellets $): 1685(\mathrm{C}=\mathrm{O}) ; 1147$ (C-N).

\section{Biological activity}

The activity against $M$. tuberculosis H37Rv (ATTC27294) was assessed using the micro plate alamar blue assay (MABA) $)^{15,16}$. This nontoxic methodology uses a thermally stable reagent, and shows a good and proportional correlation with BACTEC radiometric methods ${ }^{17,18}$.

\section{References}

1- I. E. Perepichka, D. F. Perepicka, Handbook of Thiophene-Based Materials: Applications in Organic Electronics and Photonics, 2 Volume Set, 2009, John Wiley \& Sons, Chichester, UK. S. C. Rasmussen, S. J. Evenson, C. B. McCausland. Fluorescent thiophene-based materials and their outlook for emissive applications, Chem. Commun., 2015, 51, 4528-4543.

2- L. Chan, O. Pereira, T. J. Reddy, K. D. Sanjov, C. Possion, M. Courchesne, M. Proulx, A. Siddiqui, C. G. Yannopoulos, N. Nguyen-Ba, C. Roy, D. Nasturica, C. Moinet, R. Bethell, M. Hamel, L. L'Heureux, M. David, O. Nicolas, P. Courtrmanche-Asselin, S. Brunette, D. Bilimoria, J. Bedard, Discovery of thiophene-2-carboxylic acids as potent inhibitors of HCV NS5B polymerase and HCV subgenomic RNA replication. Part 2: Tertiary amides, Bioorg. Med. Chem. Lett., 2004, 14, 797-800.

3- R. Romagnoli, P. G. Baraldi, P. Cruz-Lopez, M. Tolomeo, A. Di Cristina, R. M. Pipitone, S. Grimaudo, J. Balzarini, A. Brancale, E. Hamei, Synthesis of novel antimitotic agents based on 2-amino-3-aroyl-5(hetero)arylethynylthiophene derivatives, hetero)arylethynylthiophene derivatives, Bioorg. Med. Chem. Lett., 2011, 21, 2746-2751.

4- A. Sivadas, M. P. Satyaseela, T. Bharani, S. K. Upparapalli, N. Subbarava, Design, Synthesis, Characterization and antibacterial activity of methyl -2- (mercaptomethyl)-3-(2-thienyl) acrylate, Int. J. Pharma Sci. Res., 2011, 2, 27-35. 
5- $\quad$ S. Jain, N. Babu, S. R. Jatti, H. Sahah, S. P. Dhaneira, Synthesis, antitubercular and antifungal activities of heteroaryl-substituted oxiranes derived from Baylis-Hillman adducts, Med. Chem. Res., 2012, 21, 2744-2748.

6- S. Saeed, D. N. Rashid, N. Ali, R. Hussain, P. G. Jones, Synthesis, spectroscopic characterization, crystal structure and pharmacological properties of some novel thiophene-thiourea core derivatives, Eur. J. Chem., 2010, 1, 221-227.

7- P. R. Kumar, S. Raju, P. S. Goud, M. Sailaja, M. R. Sarma, G. O. Reddy, M. P. Kumar, V. V. R. M. K. Reddy, T. Suresh, P. Hegde, Synthesis and biological evaluation of thiophene [3,2-b] pyrrole derivatives as potential antiinflammatory agents, Bioorg. Med. Chem., 2004, 12, 1221-1230.

8- (a) W. S. Abdel-Aal, H. Y. Hassan, T. AboulFadl, A. F. Youssef. Pharmacophoric model building for antitubercular activity of the individual Schiff bases of small combinatorial library, Europ. J. Med. Chem., 2010, 45, 1098. (b) K. Bedia, O. Elçin, U. Seda, K. Fatma, S. Nathaly, R. Sevim, A. Dimoglo. Synthesis and characterization of novel hydrazide-hydrazones and the study of their structure-antituberculosis activity, Europ. J. Med. Chem., 2006, 41, 1253-1261. (c) N. P. Buu-Hoï, N. D. Xuong, N. H. Nam, F. Binon, R. Royer. Tuberculostatic hydrazides and their derivatives, J. Chem. Soc., 1953, 1358-1364. (d) K. M. Thaker, S. D. Tala, B. L. Dodiya, K. A. Joshi, K. L. Dubal, H. S. Joshi. Synthesis of oxadiazoles and pyrazolones as a antimycobacterial and antimicrobial agents, Indian J. Chem., Sect. B, 2011, 50B, 738-744.

(e) S. L. Vasoya, M. R. Patel, S. V. Dobaria, H. S. Joshi. Facile synthesis of some new azetidinones and acetyl oxadiazoles bearing benzo[b]thiophene nucleus as a potent biological active agent, Indian J. Chem. Sect. B., 2005, 44B, 405-409. (f) K. M. Thaker, V. V. Kachhadia, H. S. Joshi. Synthesis of 4-thiazolidinones and 2-azetidinones bearing benzo (b) thiophene nucleus as potential antitubercular and antimicrobial agents, Indian J. Chem. Sect B, 2003, 42B, 1544-1547.

9- M. V. N. de Souza, M. L. Ferreira, T. C. M. Nogueira, R. S. B. Golçalves, M. A. Peralta; M. S. C. Lourenço, F. R. Vicente, Synthesis and Biological Evaluation of N-(Alkyl)-2Thiophen-2-Ylacetamides Series As A New Class of Antitubercular Agents, Lett. Drug Des. Discov., 2008, 5, 221-224.

10- M. V. N. de Souza, M. C. S. Lourenço, M. A. Peralta, R. S. B. Golçalves, T. C. M. Nogueira, C. H, L. Lima, M. L. Ferreira, E. T. Silva, Synthesis and Biological Evaluation of N,N'-di(thiopheneacetyl)diamines Series as Antitubercular Agents, Phosphorus Sulfur, 2008, 183, 2990-2997.
11- M. C. S. Lourenço, F. R. Vicente, M. G. M. O. Henriques, A. L. P. Candea, R. S. B. Golçalves, T. C. M. Nogueira, M. L. Ferreira, M. V. N. de Souza, Synthesis and biological evaluation of N-(aryl)-2-thiophen-2-ylacetamides series as a new class of antitubercular agents, Bioorg. Med. Chem. Lett., 2007, 17, 6895-6898.

12- L.N. F. Cardoso, M. L. F. Bispo, C. R. Kaiser, J. L. Wardell, S. M. S. V. Wardell, M. C. S. Lourenço, F. A. F. Bezerra, R. P. P. Soares, M. N. Rocha, M. V. N. de Souza, AntiTuberculosis Evaluation and Conformational Study of $\mathrm{N}$-Acylhydrazones Containing the Thiophene Nucleus, Arch. Pharm. Chem. Life Sci., 2014, 347, 432-448.

13- A. B. Lopes, E. Miguez, A. E. Kümmerle, V. M. Rumjanek, C. A. M. Fraga, E. J. Barreiro. Characterization of Amide Bond Conformers for a Novel Heterocyclic Template of $\mathrm{N}$-acylhydrazone Derivatives, Molecules, 2013, 18, 11683-11704.

14- Y. K. C. da Silva, C. T. M. Reyes, C. G. Rivera, M. A. Alves, E. J. Barreiro, M. S. A. M. Moreira, L. M. Lima, 3-Aminothiophene-2Acylhydrazones: Non-Toxic, Analgesic and Anti-Inflammatory Lead-Candidates, Molecules, 2014, 19, 8456-8471.

15- Z. Cui, Y. Li, Y. Ling, J. Huang, J. Cui, R. Wang, X. Yang, New class of potent antitumor acylhydrazone derivatives containing furan, Eur. J. Med. Chem., 2010, 45, 5576-5584.

16- P. Patarski, E. Wyrzykiewicz, G. Bartkowiak, Synthesis and Conformational Assignment of $N$-(E)-StilbenyloxymethylenecarbonylSubstituted Hydrazones of Acetone and $o$-( $m$ - and $p$-) Chloro- (nitro-) benzaldehydes by Means of 1H and 13C NMR Spectroscopy, $J$. Spect., 2013, Article ID 197475, 12 pages.

17- V. V. Syakaev, S. N. Podyachev, B. I. Buzykin, S. K. Latypov, W. D. Habicher, I. Konovalov, NMR study of conformation and isomerization of aryl- and heteroarylaldehyde 4-tertbutylphenoxyacetylhydrazones, J. Mol. Struct., 2006, 788, 55-62.

18- G. Palla, G. Predieri, P. Domiano, C. Vignali, C. W. Turner, Conformational behaviour andE/Zisomerization of $\mathrm{N}$-acyl andNaroylhydrazones, Tetrahedron, 1986, 42, 3649-3654.

19- (a) I. Warad, S. F. Haddad, M. Al-Noaimi, B. Hammouti, T. Ben Hadda, N'-[(E)-2-Chlorobenzyl-idene]thio-phene-2-carbohydrazide, Acta Crystallogr., Sect E, 2013, 69, 1442; (b) S. Sultan, M. Taha, S. A. A. Shah, B. N. Yamin, H. M. Zaki, (E)-3-Chloro-N'-(2-fluorobenzyl-idene)thiophene-2-carbohydrazide, Acta Crystallogr., Sect E, 2014, 70, 751; (c) Y. L. Zhao, Q. -Z. Zhang, Chen, X.; M. Yu, (E)-N'-[4-(4-Chlorobenzyloxy)-3methoxybenzylidene]thiophene-2carbohydrazide, Acta Crystallogr., Sect. E, 2006, 62, 5437-5438; (d) J. -H. Jiang, 
$N^{\prime}$-[(5-Methyl-2-furyl)methylene]thiophene-2carbohydrazide, Acta Crystallogr., Sect. E, 2010, 66, 922; (e) J.-H. Jiang, $N^{\prime}$-(4-Chlorobenzylidene)thiophene-2-carbohydrazide, Acta Crystallogr., Sect. E, 2010, 66, 923; (f) Y. F. Li, F. F. Jian, $N$ '-(4-Methyl-benzyl-idene)thiophene-2-carbohydrazide, Acta Crystallogr., Sect E, 2010, 66, 1398; ( g) Y. F. Li, F. F. Jian, (E)-N'-(4-Methoxy-benzyl-idene)thio-phene-2carbohydrazide, Acta Crystallogr., Sect E, 2010, 66, 1400; (h) J. -H. Jiang, $N$ '-Benzylidenethiophene-2-carbohydrazide, Acta Crystallogr., Sect. E, 2011, 67, 50; (i) C. H. Diao, M. Yu, (E)-N'-[3-Methoxy-4-(4-nitrobenzyloxy)benzylidene]thiophene-2carbohydrazide, Acta Crystallogr., Sect. E, 2006, 62, 5441-5442; (j) Y. F. Li, F. -G. Zhang, F. F. Jian, $N$ '-(4-Cyano-benzyl-idene)thiophene-2-carbohydrazide, Acta Crystallogr., Sect E, 2010, 66, 1471.

20- (a) A. M. Alanazi, S. Lahsasni, A. A. El-Eman, S. W. Ng, N'-[(1E)-(4-Fluoro-phen-yl)methylidene]thio-phene-2-carbohydrazide, Acta Crystallogr., Sect. E, 2012, 68, 314; (b) A. M. Alanazi, S. Lahsasni, A. A. El-Eman, S. W. Ng, $\mathrm{N}^{\prime}$-[(1E)-(2,6-Difluorophenyl)methylidene]thiophene-2-carbohydrazide, Acta Crystallogr., Sect. E, 2012, 68, 315.

21- (a) H. M. Ali, S. Puvaneswary, W. J. Basirun, S. W. Ng, 3-Hydroxysalicylaldehyde 2-thienoylhydrazone, Acta Crystallogr., Sect. E, 2005, 61, 1083-1084; (b) Y. F. Li, J. -H. Jiang, F. -F. Jian, N'-(4-Hy-droxy-benzyl-idene)thiophene-2-carbohydrazide, Acta Crystallogr., Sect. E, 2010, 66, 1719; (c) Z. -L. Jing, Q. -Z. Zhang, M. Yu, X. Chen, $N$-(2-Hydroxy-3methoxybenzylidene)- $N$ '-(2thienylcarbonyl)hydrazine monohydrate, Acta Crystallogr., Sect. E, 2006, 62, 4894-4895; (d) J. -H. Jiang, $N^{\prime}$-[1-(2-Hydroxyphenyl)ethylidene]thiophene-2-carbohydrazide, Acta Crystallogr., Sect. E, 2011, 67, 32.

22- (a) Y. -F. Li, F. F. Jian, $N$ '-(3,4Dimethylbenzylidene)furan-2-carbohydrazide, Acta Crystallogr,. Sect. E, 2010, 66, 2061; (b) J. Xu, X. Hue, (E)-4-\{[2-(2-Furylcarbonyl) hydrazinylidene]methyl $\}$-2-methoxyphenyl acetate, Acta Crystallogr., Sect. E, 2011, 67, 880; (c) H.-D. Dong, Crystal structure of N'(5-chloro-2-hydroxybenzylidene)furan-2carbohydrazide monohydrate, $\mathrm{C}_{12} \mathrm{H}_{9} \mathrm{ClN}_{2} \mathrm{O}_{3} \cdot \mathrm{H}_{2} \mathrm{O}$, Z. Kristallogr. NCS, 2012, 227, 89-90; (d) J. Xu, N'-(2,6-Dichlorobenzylidene)furan-2carbohydrazide, Acta Crystallogr., Sect. E, 2012, 68, 1455; (e) H. M. Ali, S. Puvaneswary, W. J. Basirun, S. W. Ng, 3Hydroxysalicylaldehyde 2-furoylhydrazone, Acta Crystallogr., Sect. E, 2005, 61, 10791080; $(f)$ X. -S. Tai, J. Yin, M. Y. Hao, Z. -P. Liang, (E)-N'-(5-Bromo-2-

hydroxybenzylidene)furan-2-carbohydrazide monohydrate, Acta Crystallogr., Sect. E, 2007, 63, 2144-2145.

23- (a) Y. -F. Li, F. F. Jian, N'-(4-Cyano-benzylidene)furan-2-carbohydrazide monohydrate, Acta Crystallogr., Sect. E, 2010, 66, 1670; (b) Y. -F. Li, F. F. Jian, N'-Benzyl-idene-furan-2carbohydrazide, Acta Crystallogr., Sect. E, 2010, 66, 1720; (c) R. Bikas, H. H. Monfared, Kazak, C.; N. B. Arslan, K. Bijanzad, (E)-N'(2-Hydroxybenzylidene)furan-2carbohydrazide, Acta Crystallogr, Sect. E, 2010, 66, 2015; (d) Y. -F. Li, F. F. Jian, (E)- $N$ '[4-(Methyl-sulfan-yl)benzyl-idene]furan-2carbohydrazide monohydrate, Acta Crystallogr., Sect. E, 2010, 66, 2157; (e) Y. -F. Li, F. -Y. Meng, $N^{\prime}$-[4-(Dimethyl-amino)benzyl-idene]furan-2-carbohydrazide, Acta Crystallogr., Sect. E, 2010, 66, 2696; (f) J. -H. Jiang, $(E)-N^{\prime}$-(2-Fluorobenzylidene)furan-2carbohydrazide, Acta Crystallogr., Sect. E, 2010, 67, 240; ( $g$ ) A. Sundar, S. Ranjith, G. Rajagopal, $N^{\prime}$-[(E)-3-Bromo-5-chloro-2-hydroxybenzylidene]furan-2-carbohydrazide, Acta Crystallogr., Sect. E, 2014, 70, 670; (h) J. -H. Jiang, $N^{\prime}$-(4-Chlorobenzylidene)furan-2carbohydrazide monohydrate, Acta Crystallogr., Sect. E, 2010, 66, 627; (i) Y. -L. Zhao, Q. -Z. Zhang, X. Chen, M. Yu, $(E)-N^{\prime}-[4-$ (4-Chlorobenzyloxy)-3-methoxybenzylidene]furan-2-carbohydrazide, Acta Crystallogr., Sect. E, 2007, 63, 2952-2953; (j) X. -S. Tai, J. Yin, F. -Y. Z. Kong, Crystal structure of 2-carboxybenzaldehyde furan-2carbohydrazide methanol hemisolvate, $\mathrm{C}_{13} \mathrm{H}_{10} \mathrm{~N}_{2} \mathrm{O}_{4} \cdot 0.5 \mathrm{CH}_{3} \mathrm{OH}$, Kristallogr. NCS, 2007, 222, 401-402; (k) Q. -L. Zhou, C. -L. Wang, Z. -L. Jing, 2'-(1,3-Benzodioxol-5ylmethylene)furan-2-carbohydrazide, Acta Crystallogr., Sect. E, 2007, 63, 2952.

24- R. Bikas, P. M. Anarjan, S. W. Ng, E. R. T. Tiekink, $N^{\prime}-[(E)$-2-Hydroxy-5-iodobenzylidene]furan-2-carbohydrazide monohydrate, Acta Crystallogr, Sect. E, 2012, 68, 413-414. 